\title{
Interdisziplinarität im Entwurf
}

\section{Zur Geschichte einer Denkform des Erkennens in der Bundesrepublik (1955-1975)}

\author{
Susanne Schregel
}

Drafting Interdisciplinarity. Forms of Thought and Knowledge Production in the Federal Republic of Germany (19551975)

\begin{abstract}
This article traces the history of interdisciplinarity as a contemporary form of thought and of producing knowledge in the Federal Republic of Germany from 1955 to 1975. It establishes that concepts of interdisciplinary research and teaching circulated in diverse fields of knowledge and modes of articulation, and evaluates the transformations that interdisciplinarity underwent along the way. After detailing the process by which the adjective "interdisciplinary" first came into usage in scientific publications in the late 1950s, this article discusses how interdisciplinary research and teaching began to feature in debates about university reforms and the founding of new universities in the 1960s. The article then draws on the examples of Bochum, Konstanz, and Bielefeld to illustrate how debates about disciplinary specialization and interdisciplinary connections unfolded in visual modes of expression such as diagrams or sketches. In a last step, the article examines how visual and textual reflections connected interdisciplinarity to the architecture of envisioned universities, and hence related this time-specific form of thought and knowledge production to the material environments of future research and teaching.
\end{abstract}

Keywords: Circulation, cold war, epistemology, Federal Republic of Germany, history of concepts, history of knowledge, history of universities, interdisciplinarity

Schlüsselwörter: Zirkulation, Kalter Krieg, Epistemologie, Bundesrepublik, Begriffsgeschichte, Wissensgeschichte, Universitätsgeschichte, Interdisziplinarität

\section{„Interdisziplinäre" Wissenschaft: Visionen und Verwirrungen}

Wenn heute von disziplinenübergreifender Wissenschaft die Rede ist, ist die Figur der Interdisziplinarität nicht weit. Hohe Erwartungen und Begriffsverwirrungen gehen hierbei Hand in Hand. So lädt etwa die Autorin einer aktuellen Publikation zur Inter- und Transdisziplinarität zur Diskussion darüber ein, „ob interdisziplinäre und transdisziplinäre Wissenschaft Formen sein könnten, sich mehr und anders am Projekt der permanenten Wieder- und 
Neuerfindung von Gesellschaft zu beteiligen“, und verknüpft dies mit der Frage, „wie sich Wissenschaft genauer, effektiver, angemessener, kontroverser, ehrlicher, glücklicher, expliziter, radikaler und revolutionärer einmischen kann und soll“ (Winiwarter 2014: 12). Zugleich gibt der Begriff allerdings gewisse Rätsel auf. Man kann sich die Verzweiflung vorstellen, die den Verfasser eines Lexikoneintrages erfasst haben muss, wenn er in einem traditionell nach Genauigkeit strebenden Genre den Satz zu Papier bringt: „Über die allgemein akzeptierte Forderung hinaus, dass I[nterdisziplinarität] eine größere Rolle in den Wissenschaften spielen solle, gibt es wenig Klarheit darüber, was I[nterdisziplinarität] bedeutet" (Schlaeger 2008: 324).

Vielleicht mag es helfen, der Frage nach den Implikationen des Interdisziplinären nicht allein praktisch oder terminologisch, sondern auch historisch nachzugehen. Denn die Debatte um interdisziplinäre Wissenschaft ist ebenso ein Teil der Zeitgeschichte wie ein Phänomen der gegenwärtigen Wissenschaftskultur. Bereits die Begriffsgeschichte ${ }^{1}$ der Bezeichnungen „,interdisziplinär“/,Interdisziplinarität“ macht deutlich, dass es sich bei der Auseinandersetzung um Interdisziplinarität um ein Kind der jüngsten Vergangenheit handelt. ${ }^{2}$ So wurde das Adjektiv „interdisziplinär“ - in Anlehnung an US-amerikanische Vorbilder - in den 1960er Jahren in der Bundesrepublik geläufig. ${ }^{3}$ Im darauffolgenden Jahrzehnt erhob sich diese Bezeichnung eines Zwischen dann als Substantiv zu einem Sachverhalt eigener Güte ${ }^{4}$ und wurde bald als populär, ja geradezu modisch wahrgenommen. „Interdisziplinarität ist der wissenschaftsprogrammatische Slogan der 60er, sicher noch mehr der 70er Jahre", konnte ein Text zur Friedensforschung so Mitte der 1970er Jahre erklären (Brinkmann 1974: 121).

Diese Betonung des zeithistorischen Charakters der Interdisziplinarität soll nicht darüber hinwegsehen, dass Klagen über eine allzu große wissenschaftliche Spezialisierung die Herausbildung der modernen Disziplinen seit ihren Anfängen begleitet haben. ${ }^{5}$ Auch die häufig mit interdisziplinärer Wissenschaft assoziierten kooperativen und kollaborativen Praktiken gab es bereits vor dem 20. Jahrhundert (Nickelsen 2014). Das Auftreten einer neuen Bezeichnung für diese verweist aber darauf, dass es sinnvoll sein kann, Auseinandersetzungen um interdisziplinäre Wissenschaft als gegenwartsnahe Geschichte der Wissenschaft und ihrer angestrebten Erkenntnisweisen zu historisieren. Dieser Beitrag beschreibt Reflexionen über Interdisziplinarität daher als zeithistorische Ausprägungen einer bereits länger währenden Debatte um wissenschaftliche Spezialisierung und den Zusammenhang der Wissenschaften.

Die Auseinandersetzung mit Interdisziplinarität in der Bundesrepublik ist - anders als in den $\mathrm{USA}^{6}$ - noch wenig historisch orientiert. Zwar haben Soziologen, Philosophen und Wissenschaftstheoretiker versucht, Interdisziplinarität zu definieren oder interdisziplinäre Praxis zu erfassen. ${ }^{7}$ Neuere geschichtswissenschaftliche Publikationen haben das Thema zudem in den 
Kontext einer Wissens- und Ideengeschichte des Kalten Krieges gestellt (Rohstock 2013: 213-216; Bernhard et al. 2014: 31). Indem Diskussionen um disziplinenübergreifende Wissenschaft insbesondere im Zuge der Hochschulgründungs- und Hochschulreformdebatten der 1960er Jahre entstanden, finden sich Hinweise auch im Umfeld der Wissenschafts- und Universitätsgeschichte dieser Zeit. ${ }^{8}$ Allerdings fehlt bisher eine ausführliche historische Analyse, wie sich interdisziplinäre Wissenschaft in der Bundesrepublik als eine wissenschaftliche Leitvorstellung etablierte und sich dabei der Begriffe und Deutungsmuster einer international geführten Wissenschaftsdebatte bediente. Wenig wissen wir auch über den Stellenwert und die Deutungsmuster der Interdisziplinarität in der DDR, wo der Terminus ebenfalls verwendet wurde. ${ }^{9}$ Aus diesem Grund muss dieser Beitrag vorläufig bleiben; er lädt ausdrücklich zu weiteren Erkundungen und Stellungnahmen ein.

Der Beitrag versucht, erste Schritte einer Wissenschafts- und Wissensgeschichte der Interdisziplinarität in der Bundesrepublik zu unternehmen. Er möchte zeigen, wie die in den 1950er Jahren noch wenig bekannte Formel von der „interdisziplinären“ Wissenschaft seit den 1960er Jahren eine verbreitete wissenschaftliche Zielvorstellung zu markieren begann. Das Hauptinteresse liegt hierbei auf den Wissenschaftsreflexionen, die den Ruf nach fächerübergreifender Zusammenarbeit jenseits konkreter Forschungspraktiken konturierten. Denn diese Reflexionen waren es mehr noch als die von ihnen thematisierten Handlungsweisen selbst, die Interdisziplinarität als eine zeitspezifische Figur des Denkens und Erkennens begründeten.

Der Beitrag gliedert sich in vier Teilabschnitte. Diese folgen den Zirkulationen und Transformationen interdisziplinärer Wissenschaft durch unterschiedliche Wissensfelder und Artikulationsformen (Sarasin 2011; Kilcher and Sarasin 2011). Der erste Abschnitt beschreibt zunächst, wie wissenschaftliche Texte das Adjektiv „interdisziplinär“ seit den 1950er Jahren in Anlehnung an US-amerikanische Vorbilder aufgriffen. Der zweite Abschnitt führt aus, wie parallel dazu wissenschaftspolitische und wissenschaftsorganisatorische Texte der frühen 1960er Jahre die Anregung disziplinenübergreifender Zusammenarbeit zu einem Ziel von Hochschulreform und Hochschulneugründungen erklärten. Die schwerpunktmäßig diskutierten Universitäten Bochum, Konstanz und Bielefeld verpflichteten sich so ausdrücklich der Förderung disziplinenübergreifender und interdisziplinärer Kooperation. Der dritte Abschnitt wendet den Blick vom Text zum Bild; er zeigt, wie sich das Leitziel einer Verflechtung der Disziplinen im Umfeld dieser Hochschulen als visueller Entwurf entfaltete. Der vierte Abschnitt schließlich folgt sprachlichen und visuellen Denkformen des Interdisziplinären zu Imaginationen einer diese ermöglichenden Materialität; er hebt hervor, dass Texte und Bilder Konzeptionen interdisziplinärer Wissenschaft nicht zuletzt auch auf die gebauten Umwelten der Wissenschaft beziehbar machten. 
Die Auswahl der Quellen war daran orientiert, wissenschaftliche und wissenschaftspolitische Kontexte gleichermaßen zu berücksichtigen. Die Quellen sollten zudem eine sprachorientierte Analyse interdisziplinärer Wissenschaft ermöglichen (vgl. hierzu insbesondere Schauz 2014; 2015), ohne aber die Geschichte der Interdisziplinarität auf diese engzuführen. Verwendet wurden deshalb wissenschaftliche Publikationen, Zeitungs- und Zeitschriftenartikel, zentrale Dokumente des bundesdeutschen Hochschulausbaus und konzeptionelle Dokumente wie die Erklärungen der Gründungsausschüsse sowie Material zur Auseinandersetzung um die künftige räumliche und architektonische Gestaltung der genannten Hochschulen. Die Aussagen zur Nutzung der Begriffe „interdisziplinär“/,Interdisziplinarität“ in wissenschaftlichen Texten stützen sich im Wesentlichen auf die Nutzung volltextdurchsuchbarer Datenbanken. Systematisch ausgewertet wurden die Sammlungen DigiZeitschriften und jstor ${ }^{10}$ für die Jahre bis 1965 für das Suchwort interdisz* bzw. bis 1974 für das Suchwort Interdisziplinar* und damit für die Zeit, in der das Adjektiv beziehungsweise das Substantiv neu auftraten.

\section{Das Adjektiv „interdisziplinär“ in wissenschaftlichen Texten. Eine begriffsgeschichtliche Skizze}

„Interdisziplinäre“ Wissenschaft hat keinen klaren Ausgangspunkt; sie ließe sich zeitlich auf ältere Reflexionen über die Schattenseiten disziplinärer Spezialisierung rückbeziehen und ist auch räumlich kaum eindeutig auf einen Herkunftsort einzugrenzen. Gleichwohl lässt sich festhalten, dass die Geschichte der Begriffe „interdisziplinär“/„Interdisziplinarität“ nicht als deutsche Geschichte beginnt. Auch wenn diese heute ihren festen Platz in der deutschen Sprache gefunden haben, handelt es sich bei ihnen um Importe aus der US-amerikanischen Wissenschaftsterminologie. Erste Nachweise des Wortes „interdisciplinary“ finden sich in den USA der 1920er und 1930er Jahre im Umfeld des Social Science Research Councils (Frank 1988: 91-93; Sills 1986; Abbott 2001: 131-132; Moran 2010: 13). Der Terminus begann seine Karriere damit im Umfeld der amerikanischen Sozialwissenschaften.

In den 1920er und 1930er Jahren blieb das Adjektiv allerdings auch in der US-amerikanischen Wissenschaft noch eher selten und war vielen konkurrierenden Begrifflichkeiten ausgesetzt. ${ }^{11}$ Seinen Durchbruch als eine wissenschaftsnormative Figur erlebte es erst nach dem Ende des Zweiten Weltkrieges, als es sich unter den Vorzeichen des Kalten Krieges mit einem Diskurs um Offenheit und Demokratie verband und ,interdisziplinäres“ Forschen zu einer Vorgehensweise avancierte, die demokratische Tugenden wie geistige Flexibilität signalisieren wie forschend umsetzen sollte (Cohen-Cole 2014: 65-103; Frank 1988: 95-101). Seit 1950 erschien der Wortbestandteil 
„interdisciplinar*" mit zunehmender Tendenz im Titel englischsprachiger wissenschaftlicher Publikationen, wobei er in den Geistes- und Sozialwissenschaften häufiger auftrat als in den Natur- und Ingenieurwissenschaften (Larivière and Gingras 2014: 188-190).

Das Aufkommen des Wortes „interdisziplinär“ in der Bundesrepublik wird in der Regel auf die frühen 1960er Jahre datiert (vgl. etwa Reinalter 2011: 168; Hilgendorf 2010: 913; Veit-Brause 2000: 22). In seinem auch heute noch häufig zitierten Beitrag im „Historischen Wörterbuch der Philosophie“ nennt Helmut Holzhey einen wissenschaftlichen Aufsatz zur Gruppenarbeit in der Kölner Zeitschrift für Soziologie und Sozialpsychologie aus dem Jahr 1960 als ersten ihm bekannten Verwendungsnachweis (Holzhey 1976, unter Verweis auf Bahrdt et al. 1960). Mit fortschreitender Digitalisierung des deutschsprachigen Buch- und Zeitschriftenmaterials ist es heute möglich, diesen Nachweis um weitere zu ergänzen. Auf diese Weise wird deutlich, dass das Adjektiv „interdisziplinär“ in der Bundesrepublik bereits seit Mitte der 1950er Jahre vereinzelt in wissenschaftlichen Texten verwendet wurde.

Von wenigen Ausnahmen abgesehen, stand das Gros dieser frühen Belegstellen in einem direkten Zusammenhang zur US-amerikanischen Forschung und Wissenschaft. ${ }^{12}$ Wissenschaftliche Publikationen der 1950er und frühen 1960er Jahre, die das Wort ,interdisziplinär“ verwendeten, thematisierten so zum einen Formen der Wissenschaftsorganisation in den Vereinigten Staaten. ${ }^{13}$ Zudem erschien das Adjektiv in der Auseinandersetzung mit amerikanischen Forschungsarbeiten und Tagungen. ${ }^{14}$ Insbesondere deutschsprachige Rezensionen englischsprachiger Veröffentlichungen griffen das Adjektiv „interdisciplinary“ direkt aus den besprochenen Texten auf, übersetzten es und transferierten es so in die deutsche Sprache. ${ }^{15}$ Schließlich erschien das deutsche Wort „interdisziplinär“ auch in internationalen Zeitschriften, die mehrsprachig publiziert wurden - auch hier oftmals als direkte Übersetzung aus dem Englischen. ${ }^{16}$

Dieser Bezug zur US-amerikanischen Wissenschaft und Forschung lockerte sich im Verlauf der 1960er Jahre. Interdisziplinäres Arbeiten wurde in den 1960er Jahren zwar durchaus noch als „neuartiger ,approach’ für die wissenschaftliche Forschung "17 wahrgenommen, und das Adjektiv erschien in wissenschaftlichen Texten kombiniert mit der Bezeichnung „international“ ${ }^{18}$ Dennoch wurde es allmählich zu einem Teil der bundesdeutschen Wissenschaftssprache. Dies wird etwa deutlich, wenn ein Tagungsbericht 1964 von „interdisziplinär“ (in Anführungszeichen) als „dem beliebt gewordenen neuen terminus“ (Schiffers 1964: 33) spricht. 1965 bezeichnete ein Wissenschaftler in einem Vortrag „die Wortbildung ,interdisziplinär"“ als „heute international durchgesetzt" (Göppinger [1965] 1966: 1). 


\section{Disziplinenübergreifende Forschung und Lehre in wissenschaftspolitischen und wissenschaftsorganisatorischen Debatten}

Das Adjektiv „interdisziplinär“ verbreitete sich damit in wissenschaftlichen Texten parallel zu umfassenderen Veränderungen der bundesdeutschen Hochschullandschaft. So hatte die Verdopplung der Studierendenzahlen zwischen 1955 und 1965 (Rohstock 2010: 25) zu einem erhöhten Druck auf die bestehenden Universitäten geführt und den Wunsch nach Neugründungen mit Entlastungsfunktion entstehen lassen. Zugleich entflammte in den 1960er Jahren eine Debatte über wissenschaftlich-universitäre Reformen, welche sich besonders auf die neu entstehenden Universitäten richteten. ${ }^{19}$ In dieser Situation speiste und formte ein Krisendiskurs um eine zunehmende wissenschaftliche Zersplitterung durch Wachstum und Spezialisierung Forderungen nach intensivierter disziplinenübergreifender Kooperation in Forschung und Lehre. $^{20}$ Klagen über eine sich zersplitternde und ihre „Einheit“ verlierende Wissenschaft in den Zeiten der Massenuniversität trieben so die Suche nach Hochschulkonzepten an, die diesen Tendenzen entgegenwirken könnten.

Die Forderung nach disziplinenübergreifender Kooperation zog sich gleichsam leitmotivisch durch die „Anregungen des Wissenschaftsrates zur Gestalt neuer Hochschulen“ (1962), einem Schlüsseldokument des bundesdeutschen Hochschulausbaus (Anregungen 1962). Die „Anregungen“ diagnostizierten eine zunehmende Ausdifferenzierung wissenschaftlicher Disziplinen; diese begründete die Forderung, fächerübergreifende Kooperationen zu ermöglichen, um den Zusammenhalt der Wissenschaft zu stärken. Der Wissenschaftsrat stellte zunächst einen Prozess der wissenschaftlichen Ausdifferenzierung fest, der nicht durchweg negativ konnotiert erschien; Ausdifferenzierung und Spezialisierung seien notwendigerweise erfolgt und parallel zum „Fortschritt der Wissenschaft“ verlaufen (ebd. Nr. 12). Vielmehr waren es die Konsequenzen der Spezialisierung, welche die „Anregungen“ als negativ beschrieben. Indem sich nämlich die wissenschaftlichen Teilgebiete ausfächerten, schien die „Einheit des Ganzen“ (ebd. Nr. 12a) gefährdet. Denn die Spezialisierung habe eine „Verselbstständigung der Teile“ (ebd. Nr. 18) der Universität begünstigt. Nicht weiter integriert, entwickele die Universität zentrifugale Tendenzen (ebd. Nr. 12a.). An die Stelle von Wissenschaft als einer ursprünglichen und durch die Universitäten vertretenen Einheit rückte so das Schreckbild eines zersplitterten, sich in seinen Eigendynamiken verfangenden Gemenges isolierter Teilwissenschaften.

Fächerübergreifende Kooperation schien demgegenüber geeignet, die konstatierte Zerfaserung und Zersplitterung von Wissenschaft und Universität aufzufangen. Da die „Idee der Vollständigkeit“ ohnehin nicht mehr verwirklicht werden könne, hielt es der Wissenschaftsrat stattdessen für 
ratsam, „eine fruchtbare Zusammenarbeit zwischen verschiedenen Fächergruppen zu ermöglichen“ (ebd. Nr. 13). Dementsprechend empfahl er etwa die Neueinrichtung „zentraler Institute“ mit der Aufgabe, „den durch die Spezialisierung gefährdeten Zusammenhang größerer Fachgebiete innerhalb einer Fakultät und über Fakultätsgrenzen hinaus wieder in gemeinsamer Arbeit herzustellen oder die Methoden und Ergebnisse verschiedener Disziplinen zur Lösung gemeinsamer, die Fachgrenzen übergreifender Probleme fruchtbar zu machen“ (ebd. Nr. 15). Ferner sei die bereits gegebene „Ordnung der Fächer" daraufhin zu prüfen, ob innerhalb der traditionellen Gliederungen „Verbindungen zu benachbarten Fächergruppen“ hergestellt werden könnten (ebd. Nr. 13). Unter anderem zum Abbau von Animositäten zwischen den Disziplinen regte der Wissenschaftsrat an, auch die Ingenieurwissenschaften in die Universitäten mit einzubeziehen (ebd. Nr. 56).

Die Befürwortung fächerübergreifender Zusammenarbeit stellte sich in den Anregungen des Wissenschaftsrates somit nicht zuletzt als eine kompensatorische Anstrengung dar; sie plausibilisierte sich als das Bemühen, eine einstmals vorhandene Verbindung zwischen den Wissenschaftsdisziplinen zu „bewahren“ oder zumindest wissenschaftsnormativ zu kompensieren. Aus der eigenen Disziplin hinauszublicken wurde zur wissenschaftsmoralischen Verpflichtung gegenüber einer Vision von Wissenschaft als übergeordneter Ganzheit; fächerübergreifende Kooperation avancierte zum Fluchtpunkt einer mereologischen Verlusterzählung, in deren Folge es Teil und Ganzes der Wissenschaft neu zu bestimmen galt. $^{21}$

Anlehnend an die Überlegungen des Wissenschaftsrates, erlangte das Thema der disziplinenübergreifenden Zusammenarbeit in den Gründungsprozessen neuer Universitäten besondere Aufmerksamkeit. Insbesondere bei den Hochschulneugründungen Bochum, Konstanz und Bielefeld nahm das Ziel einer vermehrten Kooperation und Verbindung zwischen den wissenschaftlichen Teilbereichen einen hohen Stellenwert ein. So machte der Gründungsausschuss der Universität Bochum 1962 die Förderung einer ,allseitige(n) Verflechtung der wissenschaftlichen Disziplinen“ zur Grundlage seiner Überlegungen über die Gestalt einer künftigen Hochschule. ${ }^{22}$ In der Denkschrift zur Gründung der Universität Konstanz vom Juni 1965 war den angestrebten „Formen der Kooperation“ gar ein gesondertes Kapitel gewidmet. Dies sollte das „Gewicht, das der Gründungsausschuß der Kooperation in Konstanz zumißt", hervorheben. ${ }^{23}$ Auch in Bielefeld bildete die Ermöglichung disziplinenübergreifender Kommunikation und Kooperation ein Kernstück der Hochschulkonzeption. ${ }^{24}$

Im Umfeld dieser Gründungsdiskussionen wurde nun auch das Adjektiv „interdisziplinär“ verwendet. Während die Empfehlungen zur Gründung der Universität Bochum von 1962 noch eher Formulierungen wie „Verflechtung“ und "Ganzes“ der Universität nutzten (Empfehlungen Bochum [1962] 1968: Nr. 392; Nr. 396), erschien drei Jahre später in Konstanz das Attribut 
„interdisziplinär" sporadisch. Die eigentliche Betonung in den Empfehlungen des Gründungsausschusses lag hier zwar auf dem Begriff der „Kooperation“ in Absetzung vom ,team work“, das im Vergleich dazu stärker auf die Lösung eines einzelnen Problems ausgerichtet sei (Die Universität Konstanz [1965] 1968: Nr. 1432-1442). Der Gründungsausschuss hob aber hervor, dass in der Bildung von Gruppen für die wissenschaftliche Arbeit „ein so wichtiges und für das Gedeihen der interdisziplinären Forschung unentbehrliches Element“ liege, dass es bei der Berufung von Professoren für die neue Universität bedacht werden müsse (ebd. Nr. 1435). Im Anschluss an die Skizzierung von Möglichkeiten kooperativer Forschung formulierte der Gründungsausschuss zudem die Erwartung, „daß bei der beschränkten Studentenzahl auch in der Lehre die Wirkung dieser interdisziplinären wissenschaftlichen Arbeit spürbar wird und damit die Studenten sich nicht nur im Horizont ihre Fächer bewegen“ (ebd. Nr. 1435). Darüber hinaus wandte er sich dagegen, „daß für Problemkreise oder Fachkomplexe, die ihrer Natur nach nur interdisziplinär zu bearbeiten sind, eigens Lehrstühle eingerichtet werden, die dann nur einem Teil des Ganzen gerecht werden“" (ebd. Nr. 1441).

In den Gründungsdokumenten der Universität Bielefeld erschien die Bezeichnung ,interdisziplinär“ seit Mitte der 1960er Jahre an zentraler Stelle und vielfach wiederholt. ${ }^{25}$ Nach der Denkschrift Helmut Schelskys zu den „Grundzüge(n) einer neuen Universität“ aus dem Jahr 1965 lagen in „(i)nterdisziplinäre(r) Forschung“ beziehungsweise in „interdisziplinärer Zusammenarbeit" (Schelsky [1965] 1966b: 42-44) erhebliche Potenziale für den Fortgang der Wissenschaft: „Interdisziplinäre Forschung verschiedenster Art gehört heute $\mathrm{zu}$ den entscheidenden Grundlagen wissenschaftlichen Fortschritts und ist institutionell in die Hochschulen einzubauen" (ebd. 38). Statt „Dauerinstitute für interdisziplinäre Forschungsaufgaben“ zu schaffen, welche „die zeitweilig fruchtbaren Fächerkombinationen zu einer Dauerspezialisierung erhebt und erstarren läßt“ (ebd. 43), regte Schelsky die Festlegung von „Universitätsthematiken“ als „Grundlage interdisziplinärer Zusammenarbeit" an und schlug vor, „interdisziplinäre Forschungsprojekte“ zu verabreden (ebd. 42-44).

Die „Re-Integration“ der Disziplinen sollte zusätzlich durch die Einrichtung eines speziellen Forschungsinstitutes, des „Zentrums für interdisziplinäre Forschung", angeregt werden (ebd.). Vorbilder für eine solche Einrichtung sah Schelsky sowohl in der europäischen als auch in der nordamerikanischen Wissenschaftstradition. ${ }^{26}$ Die Notwendigkeit eines Zentrums für interdisziplinäre Forschung resultierte für Schelsky nicht zuletzt auch aus den politischen Entscheidungen zum Hochschulausbau der vorhergegangenen Jahre, wenn er in der Denkschrift zur Gründung des ZiF erklärte: „Der energische Ausbau der Hochschulen in den letzten Jahren hat die schon verkümmerte Kommunikation zwischen den Fächern noch erheblich erschwert, weil er nur die Spezialisierung vertiefte“ (Schelsky 1966a: 74). 
Demgegenüber sollte das Zif eine „Re-Integration der sich spezialisierenden Wissenschaften" als zeitweilige "Zusammenarbeit" unterschiedlicher Disziplinen ermöglichen (ebd. 72). Diese Zusammenarbeit stellte Schelsky als temporär und themengebunden heraus (ebd.).

Diese Beispiele zeigen, wie disziplinenübergreifende Zusammenarbeit zu einem wichtigen Thema in den Debatten um die Gründung neuer Hochschulen wurde. Damit gelangte das Adjektiv „interdisziplinär“, das auch bereits zuvor in wissenschaftlichen Texten verwendet wurde, in wissenschaftspolitische und wissenschaftsorganisatorische Zusammenhänge. Parallel dazu begann sich das Adjektiv auch in wissenschaftlichen Publikationen stärker direkt auf die Bundesrepublik zu beziehen. So belegten wissenschaftliche Texte und Notizen in wissenschaftlichen Zeitschriften seit den frühen 1960er Jahren vermehrt bundesdeutsche Forschungsprojekte sowie Bildungs- und Forschungseinrichtungen in der Bundesrepublik mit dem Attribut „interdisziplinär“. ${ }^{27}$ Zudem erschien das Adjektiv seit den 1960er Jahren auch in einer allgemeineren Öffentlichkeit, mit deutlich zunehmender Tendenz zum Ende des Jahrzehnts. ${ }^{28}$

Reflexionen interdisziplinärer Wissenschaft blieben also nicht auf ein Wissensfeld beschränkt, sondern zirkulierten in unterschiedlichen Kontexten und Zusammenhängen. Dies verdeutlicht, dass es nicht einige wenige „Vordenker" im Umfeld der Hochschulgründungs- und -reformdebatten allein waren, die das Adjektiv in der Bundesrepublik bekannt machten. Eher geschah dies im Zuge eines breiteren Prozesses, in dessen Verlauf Wissenschaftler, Wissenschaftsorganisatoren und Politiker ihre Annahmen über die Zusammenarbeit und den Zusammenhang der Wissenschaft unter den Leitstern des interdisziplinären Arbeitens stellten, und dadurch eine insgesamt ältere Auseinandersetzung um die Einheit und den Zusammenhang der Wissenschaften unter einer neuen Begrifflichkeit zeitspezifisch variierten.

\section{Die „Verflechtung der Disziplinen“ als visueller Entwurf: Bochum, Konstanz, Bielefeld}

Die hochschul- und wissenschaftspolitischen Neuansätze der 1960er Jahre sind bereits Gegenstand vieler Publikationen geworden. Abgesehen von architekturhistorischen Arbeiten und reich bebilderten Festschriften, gehen die meisten geschichtswissenschaftlichen Arbeiten zum Thema allerdings stark sprachbasiert vor; sie gehen wenig darauf ein, inwiefern sich die Suche nach der Gestalt künftiger Wissenschaft und Hochschulen und der mit diesen verbundenen Erkenntnisweisen auch in der Visualität vollzog.

Tatsächlich war die Wissenschafts- und Hochschuldebatte der 1960er und 1970er Jahre auf den ersten Blick sprachlich dominiert: Parlamentarier 
und die Mitglieder der Gründungsausschüsse setzten sich in mündlicher Rede mit der Gestalt künftiger Hochschulen auseinander; Intellektuelle publizierten umfängliche Schriftwerke zum Thema, die oftmals ohne ein einziges Bild auskamen. Doch spätestens, als in den frühen 1960er Jahren die konkrete Planung neu zu errichtender Hochschulen begann, wurden auch Bilder zu einem Medium der Wissenschaftsreflexion. Das Beispiel der Universitätsneugründungen Bochum, Konstanz und Bielefeld zeigt, dass sowohl die Vertreter der Gründungsausschüsse als auch die politischen Gremien Diagramme, Schemata, Skizzen und Pläne nutzten, um sich über Formen und Wege von Wissenschaft und wissenschaftlichem Erkennen zu verständigen.

Diese Bilder von Wissenschaft unterscheiden sich von den Bildern der Wissenschaft, die in der wissenschaftsgeschichtlichen Forschung bisher die meiste Aufmerksamkeit erhalten haben. Denn bei ersteren ging es nicht vordringlich darum, Dinge in einem produktiven Sinne sichtbar zu machen. ${ }^{29}$ Imaginativ und vorwärtsgerichtet, entwickelten sie vielmehr Konzeptionen von Wissenschaft und wissenschaftlicher Erkenntnis, die teils erst in der Folge einen materiellen oder praktischen Bezug erhalten konnten. Da die im Folgenden betrachteten, ansonsten durchaus unterschiedlichen Bilder diese Eigenschaft teilen, werden sie hier als „Entwürfe“ zusammengefasst. $^{30}$

Text und Bild bilden hierbei keinen Gegensatz; im Gegenteil stützten sie sich wechselseitig und konnten auch direkt auseinander hervorgehen. Dies soll eingangs durch zwei Beispiele aus dem Umfeld der Entstehung der Universität Bochum hervorgehoben werden.

Abbildung 1 zeigt die „Struktur der Universität“ genannte Zusammenstellung geplanter „Abteilungen“ und Einrichtungen. Mit diesem Strukturplan machte der Gründungsausschuss im Dezember 1962 einen Vorschlag über Zahl und Ausgestaltung der geplanten universitären Teilbereiche. Der Plan könnte auch als Auflistung charakterisiert werden und ist stark textlich geprägt. Der Text ist linksbündig aufeinander orientiert, während die zugeordneten Zahlen links nicht-bündig angeordnet sind.

Der "Struktur der Universität Bochum“ ging in den Empfehlungen des Gründungsausschusses eine „Schematische Darstellung der räumlichen Zuordnung der einzelnen Abteilungen zueinander" voran (siehe Abb. 2). Diese präsentierte die geplanten Abteilungen der Universität als sechs separat angeordnete Textblöcke, welche sich um ein mittiges Rechteck der „Zentralstellen" gruppierten.

Dieses Aneinanderrücken von Text zu Textblöcken konstituierte eine Form der Bildlichkeit, in deren räumlicher Anordnung „Bild-“ und „Sprachcharakter" des Textes ineinander übergehen und eine Form der Sprachlichkeit entwickeln, die räumlich ist (Krämer 2009). Die Darstellungsweise erzeugte räumliche Beziehungen zwischen den textlichen Bezeichnungen der geplanten Abteilungen, die ebenso als Formulierung inhaltlicher Nähe- und Distanzbeziehungen zu verstehen sind. Auf diese Weise legte das Schema 
visuell eine Gruppierung von Disziplinen nahe, an denen sich das vom Gründungsausschuss der Universität Bochum formulierte Ziel einer ,allseitige(n) Verflechtung der wissenschaftlichen Disziplinen" (Empfehlungen Bochum [1962] 1968: Nr. 392) möglicherweise umsetzen ließe.

Die Beispiele in Abb. 1 und 2 deuteten die Beziehungen der Disziplinen zueinander durch die räumliche Anordnung von Text und Zahlen nur an. Dennoch entfalteten bereits diese eher reduzierten Beispiele Vorstellungen über das Verhältnis der Disziplinen und adäquate Praktiken der Wissensgenerierung; sie umrissen Konzeptionen wissenschaftlichen Erkennens und fächerübergreifender Kooperation durch die räumliche Anordnung von Textstücken. Andere Bilder aus dem Umfeld der Hochschulneugründungen griffen die Relationen der Disziplinen in expliziterer Form auf. Ausgeführt als Linie und Kreuzung von Linien, tritt uns in diesen das Leitziel einer „Verflechtung" der Disziplinen als visueller Entwurf entgegen.

So enthielt die Denkschrift des Kultusministeriums über die Errichtung von wissenschaftlichen Hochschulen in Baden-Württemberg aus dem Jahr 1962 ein „Strukturschema“ der Universität Konstanz (siehe Abb. 3).

Um ein klar umrissenes, kreisförmiges „Zentrum“ gruppierten sich zahlreiche Kreise, die Bezeichnungen wissenschaftlicher Disziplinen trugen. Die verschieden großen - Kreise waren durch Linien untereinander und zum Zentrum hin verbunden; einige nicht beschriftete und nicht verbundene Kreise deuteten den unabgeschlossenen, noch der Ergänzung bedürftigen Charakter der Darstellung an.

In diesem „Strukturschema“ evozierten die Verbindungslinien zwischen den Fächern die Vorstellung disziplinenübergreifender Kommunikation und Kooperation. Hierbei rekurrierten einerseits geschlossene Kreise auf die einzelnen Disziplinen. Andererseits blieb ihre Abschließung aber unvollkommen, indem die einzelnen Kreise durch Linien zu anderen Disziplinen durchkreuzt wurden. Die Darstellungsform könnte im weitesten Sinne als eine solche des Netzwerkes beschrieben werden; sie brachte durch ein Geflecht aus sich überkreuzenden Linien die Zielvorstellung von notwendig isolierten, gleichwohl aber auf Austausch angewiesenen wissenschaftlichen Disziplinen zum Ausdruck.

Eine weitere Facette visuell gestützter Wissenschaftsreflexionen lässt sich am Beispiel dreier Skizzen erkunden, die im Umfeld des Gründungsausschusses der Universität Konstanz entstanden (Abb. 4). Dieses im März 1964 eingesetzte Gremium legte im Juni 1965 seinen Bericht vor, der die Universität als eine Forschungsuniversität mit höchstens 3.000 Studierenden und etwa 100 Lehrstühlen konzipierte (Paulus 2010: 515-520). Die drei Zeichnungen erschienen in genau dieser Anordnung in der Baudokumentation aus dem Jahr 1970. Den Skizzen wurde also zeitnah zu ihrer Entstehung eine gewisse Relevanz zugewiesen. 
Struktur der Universität
I. Evangelisch-Theologische Abteilung
II. Katholisch-Theologische Abteilung
III. Abteilung für Philosophie, Pädagogik und Psychologie
IV. Abteilung für Geschichtswissenschaft

V. Abteilung für Sprach- und Literaturwissenschaften

VI. Rechtswissenschaftliche Abteilung

VII. Wirtschaftswissenschaftliche Abteilung

VIII. Sozialwissenschaftliche Abteilung

IX. Abteilung für Maschinenbau und konstruktiven Ingenieurbau

X. Elektrotechnische Abteilung

XI. Mathematische Abteilung

XII. Physikalische Abteilung

XIII. Geowissenschaftliche und Astronomische Abteilung

XIV. Chemische Abteilung

XV. Biologische Abteilung

XVI. Naturwissenschaftlich-Medizinische Abteilung

XVII. Theoretische Medizinische Abteilung

XVIII. Praktische Medizinische Abteilung

XIX. Allen Abteilungen zugeordnete Institutionen 
Schematische Darstellung der räumlichen Zuordnung der einzelnen Abteilungen zueinander

I. Evangelisch-Theologische Abteilung

II. Katholisch-Theologische Abteilung
III. Abteilung für Philosophie, Pädagogik und Psychologie

IV. Abteilung für Geschichtswissenschaft

V. Abteilung für Sprachund Literaturwissenschaften
XVI. Natur-

wissenschaftlichMedizinische Abteilung

XVII. Theoretische Medizinische Abteilung

XVIII. Praktische Medizinische Abteilung

\section{Zentralstellen}

Rektorat, Senat usw.

Universitätsbibliothek

Hörsaalgebäude

Hauptmensa
VI. Rechtswissenschaft= liche Abteilung

VII. Wirtschaftswissenschaftliche Abteilung

VIII. Sozialwissenschaftliche Abteilung

\footnotetext{
XI. Mathematische Abteilung

XII. Physikalische Abteilung

XIII. Geowissenschaftliche und Astronomische Abteilung

XIV. Chemische Abteilung

XV. Biologische Abteilung
} 


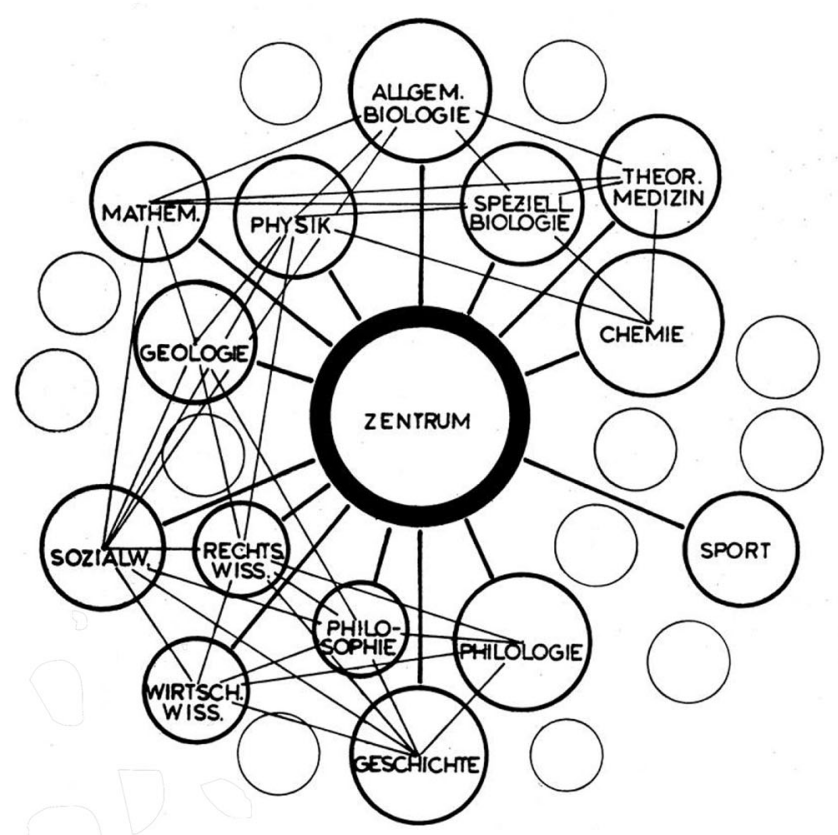

\section{STRUKTURSCHEMA}

Abb. 3 Strukturschema Konstanz (Kultusministerium Baden-Württemberg 1962: Karte Nr. 9)

Die „Schemaskizze von Prof. Besson“ oben links trägt den Titel „Das grosse Haus der Wissenschaften“. Unten links sehen wir ein „Zuordnungsschema der Zentralbibliothek“. Die Zeichnung oben rechts trägt die Bildunterschrift "Grundstein der Universität Konstanz auf dem Giessberg“. Die drei Skizzen unterschieden sich also in ihrem Gegenstandsbereich recht stark voneinander; sie referierten auf die künftige Universität als Ganzes, den Grundstein der Universität als symbolische Repräsentation sowie den möglichen Aufbau der Bibliothek. Die Zusammenfassung der Skizzen zu einem Ensemble kann also kaum durch ihren Gegenstandsbereich erklärt werden. 


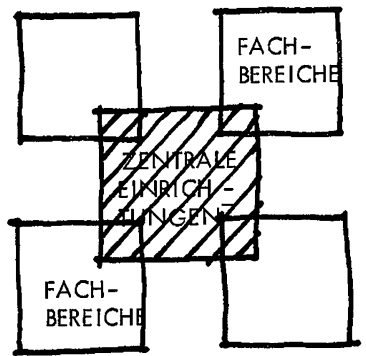

SCHEMASKIZZE VON PROF. BESSON DAS GROSSE HAUS DER WISSENSCHAFTEN
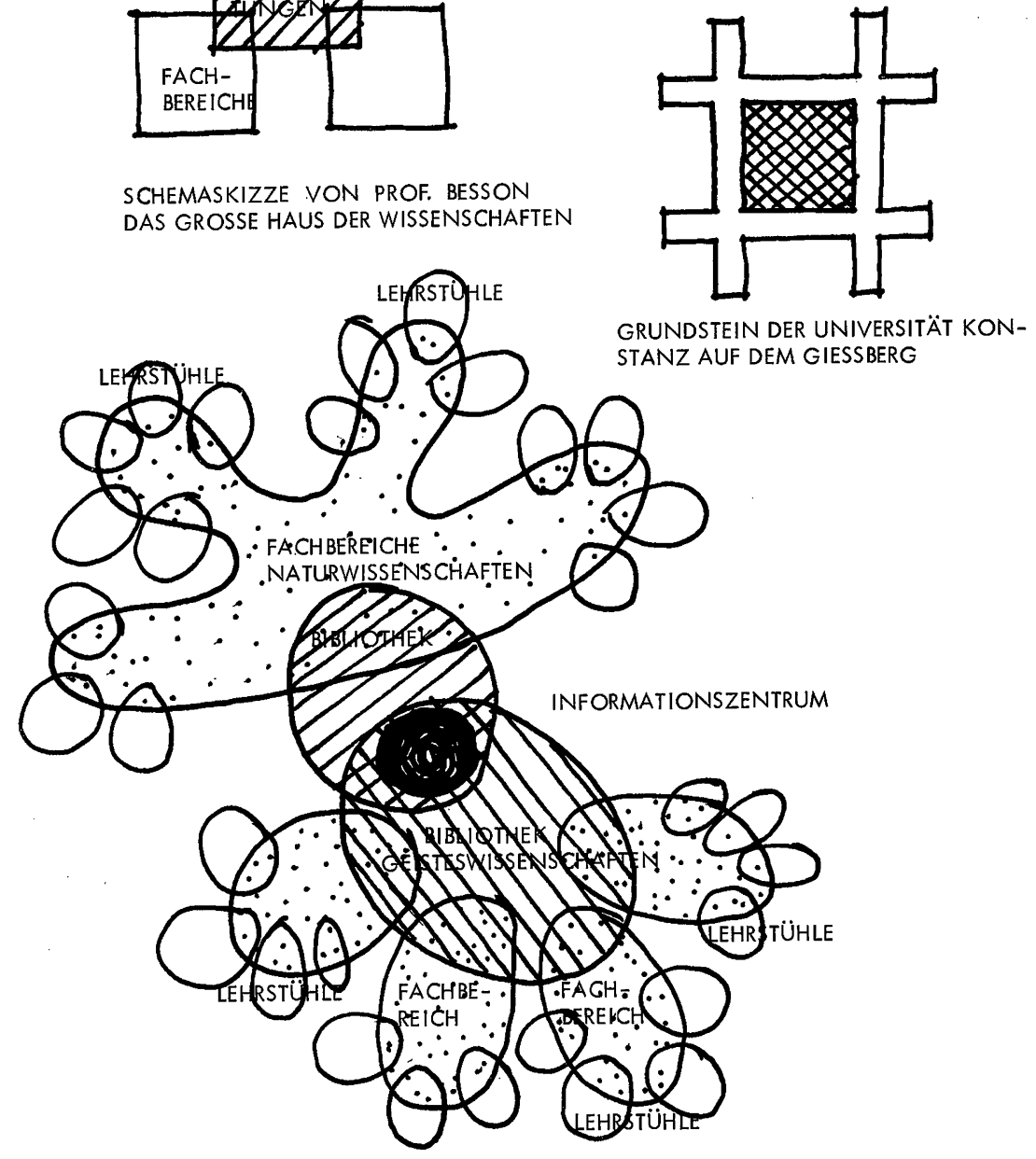

ZUORDNUNGSSCHEMA DER ZENTRALBIBLIOTHEK

Skizzen aus der Beratungszeit des Gründungsausschusses

Abb. 4 Skizzen des Gründungsausschusses Konstanz (von Mann 1970: 25)

Um die Gruppierung der Skizzen besser zu verstehen, müssen wir sie im Detail studieren.

Die Zeichnung oben links besteht aus vier unter einem zentralen Quadrat angeordneten Quadraten. Das mittig angeordnete größte Quadrat wurde über die vier äußeren gelegt und überschnitt diese; die Schraffur des inneren 
Quadrates bildete eine weitere Form der Überkreuzung. Die Zeichnung oben rechts kann als gefüllte Gitterstruktur beschrieben werden. Ihre äußeren Linien waren selber als Gitternetz angelegt. Die Fläche, die durch die Überkreuzung dieser Gitternetzlinien gebildet wurde, erschien schraffiert, wobei sich die Linien hier diagonal in zwei Richtungen durchkreuzten. Das Zuordnungsschema der Bibliothek als untere Zeichnung schließlich zeigte einen komplexeren Zusammenhang ovaler Flächen, die einander überschnitten und Schnittmengen bildeten; neben diagonalen Schraffuren deuteten in diesem Fall auch Punkte Flächen der Überschneidung an. Dies legt nahe, den Grund für die Zusammenfassung der Skizzen in ihrer formalen Ähnlichkeit zu suchen. Denn weniger als ein konkreter Gegenstand scheint es das Motiv der Überlappung, Überkreuzung und Verbindung selber $\mathrm{zu}$ sein, welches die Bilder als gestaltendes Element aneinander band.

Die betrachteten Bilder formulierten damit nicht einfach Sachverhalte, die ebenso gut sprachlich hätten ausgedrückt werden können. Sie leisteten mehr noch einen eigenständigen Beitrag zur Konstitution von Epistemologien. Denn sie bildeten ein Repertoire an Formen aus, in denen die Modi interdisziplinärer Kommunikation und Kooperation imaginiert und plausibilisiert werden konnten. Die Bildlichkeit ermöglichte zudem eine gedankliche Übertragung, welche in der Schriftlichkeit oder Mündlichkeit nicht in dieser Art und Weise möglich gewesen wäre. Überschneidungen in ihrer raumbildenden Funktion hervorhebend, machte sie die Überkreuzung von Linien in doppelter Weise deutbar - nämlich zum einen als Schnittpunkt zwischen den wissenschaftlichen Disziplinen, und zum anderen als Gestalterin eines Raumes wissenschaftlicher Kooperation.

\section{Ermöglichende Materialität: Imaginationen interdisziplinärer Wissenschaft als Kommunikation und Kooperation an einem ort}

Indem Bilder Raum- und Wissenschaftsreflexionen aufeinander beziehen konnten, trugen sie zugleich dazu bei, postulierte Formprinzipien der Wissenschaft mit der Architektur der neuen Hochschulen zusammenzubringen. ${ }^{32}$ In Auseinandersetzungen um die räumlich-architektonische Gestaltung der neuen Hochschulen wurde die Figur der Grenzüberschreitung (zwischen den Disziplinen) über Visualisierungen sich kreuzender Linien, Schnittmengen und raumbildender Überschneidungen so von einer abstrakten Relation hin zu einer Konzeption alltäglicher Wissenschaftspraxis in die Materialität gebauter Umwelten der Wissenschaft fortgeführt. Die Denkform interdisziplinärer Wissenschaft war damit einerseits beweglich und wanderte durch verschiedene Wissensfelder; andererseits wurde sie aber auch an konkrete Orte der Wissenschaft gebunden, in denen sich Zielvorstellungen 
disziplinenübergreifender Kooperation und Kommunikation lokal verankerten und materiell verdichteten. ${ }^{33}$ In der Folge dieses Prozesses wurde auch Hochschulgebäuden die Fähigkeit zugeschrieben, disziplinenübergreifende Wissenschaft zu ermöglichen und auf diese Weise selber zu „Objekten des Wissens“ zu werden, ,an denen der Prozess der wissenschaftlichen Erkenntnisgewinnung in Gang gesetzt sowie in Gang gehalten wird" (Rheinberger 2007: 10 f.).

Wie die eingangs betrachteten Reflexionen des Wissenschaftsrates, war auch die Suche nach einer adäquaten baulichen Form künftiger Hochschulen von Warnungen vor einer Zersplitterung der Wissenschaft und dem Streben nach Gegenmaßnahmen geprägt. So forderte etwa die Ausschreibung des architektonischen Ideenwettbewerbs der Universität Bochum aus dem Jahr 1962, die neue Universität solle „entsprechend den heutigen Forschungsaufgaben eine vielfältige Zusammenarbeit der verschiedenen Wissenschaftszweige, die sich immer stärker voneinander getrennt haben, ermöglichen und angesichts der fortschreitenden Spezialisierung in gemeinsamen Einrichtungen und Veranstaltungen den Gedanken der ,Universität' im eigentlichen Sinne erneut sichtbar machen. “34

Der baulichen Form der Universität wurde im Bielefelder Fall die Möglichkeit zugeschrieben, fächerübergreifende Kooperation nicht nur zu symbolisieren, sondern auch praktisch Einfluss auf die Gestaltung von Lehre und Forschung zu nehmen. Schließlich werde wissenschaftliche Arbeit, so der Rektor der Universität Bielefeld, als „hochempfindlicher Kommunikationsprozeß [...] von einem Gebäude nicht nur beherbergt, sondern auch in sich strukturiert" (Grotemeyer 1975: 7).

Die Ermöglichung disziplinenübergreifender und kooperativer wissenschaftlicher Praxis wurde folgerichtig zu einem Ziel und Gütesiegel auch architektonischer Formfindungsprozesse. Entwürfe und Planungen um die Errichtung neuer Hochschulen kreisten um die Frage, wie die bauliche Gestalt der neuen Hochschulen eine fächerübergreifende Kooperation befördern könnte; Entwürfe universitärer Architekturen strebten danach, eine Gestaltung $\mathrm{zu}$ finden, welche den Zusammenhang wissenschaftlicher Einzeldisziplinen betonen und durch materielle Vorgaben auch praktisch befördern könnte. Auf diese Weise artikulierte sich die Auseinandersetzung um die Gestalt künftiger Wissenschaft und die Ermöglichung disziplinenübergreifender Kooperation auch in der Debatte um die baulicharchitektonische Gestaltung künftiger Hochschulen. ${ }^{35}$

In diesem Versuch, eine Zusammenarbeit unterschiedlichster Wissenschaftszweige zu ermöglichen, kam dem Motiv der räumlichen Nähe eine große Bedeutung zu. ${ }^{36}$ Architekturhistorisch gesehen, knüpfte diese Wertschätzung von räumlicher Nähe und Integration für die wissenschaftliche Arbeit an US-amerikanische und britische Campus- und College-Konzeptionen an (Muthesius 2000: 203-246; Hallauer 1990: 214-16). 
Wissenshistorisch betrachtet, begründete sie sich jedoch zugleich auch durch Deutungen, welche auf die Ermöglichung von Kommunikation und Kooperation zwischen den Disziplinen abzielten. So hob etwa das Preisgericht zum Bauwettbewerb für die Universität Bielefeld in seiner Stellungnahme hervor, dass das Ziel der Kommunikation durch eine räumliche Integration des zu entwickelnden Hochschulgebäudes gefördert werden könne. Kommunikation sei zwar „mehr zufälliger Natur und nur in selteneren Fällen durch organisierte Tätigkeit oder Themata bestimmt. "Aber:

Sie kann Verursacher der Kooperation sein. Kommunikation wird durch ein entsprechendes bauliches und räumliches Angebot provoziert und begünstigt. Insofern ist nicht so sehr für die Kooperation als insbesondere für die Kommunikation eine enge Beziehung der Bereiche untereinander anzustreben (Gutachtliche Stellungnahme 1969).

Das Preisgericht forderte daher „einen räumlich gegliederten, im ganzen aber zusammenhängenden Baukomplex der Universität, um die notwendige Kooperation und Kommunikation zu gewährleisten. Denn ein „völliges Auseinanderreißen der einzelnen Fachbereiche und ihre Dezentralisierung im Universitätsgebiet oder gar im Stadtgebiet würden die Kommunikation unmöglich machen und die Kooperation in nicht zumutbarem Maße erschweren.“ Besondere Bedeutung maß das Preisgericht hierbei „Kontaktgelegenheiten“ im Sinne von „Kontaktzonen für Kooperation und insbesondere Kommunikation“ zu (ebd. 31).

Allerdings fanden baulich-gestalterische Imperative wie diese nicht auf direktem Wege zu einer "Sichtbarmachung" in einem universitären Gebäudekomplex. Erst über Bilder und Modelle vermittelten sich normative Vorgaben wie die oben zitierten auf konkrete Baustrukturen. Zeichnerisch entworfene Formen dienten zudem als Reflexionsgrundlage über den Zusammenhang von räumlichen Strukturierungen und interdisziplinärer Kooperation; sie reflektierten Möglichkeitsbedingungen von Kommunikation und Kooperation und ergründeten diese in der Gestaltung architektonischer Formen.

Dieser Gedanke soll hier anhand zweier Beispiele aus der Gründungsphase der Universität Bielefeld erläutert werden. Abbildung 5 zeigt ein Diagramm aus der Wettbewerbsdokumentation für den Bau der Universität Bielefeld (Herzog et al. 1969: 39); Abbildung 6 einen Ausschnitt aus der Bauplanungsdokumentation. ${ }^{37}$

Das obere Diagramm in Abbildung 5 stellt ein kreisförmig ausgerichtetes Netzwerk dar - nach der beigefügten Erläuterung ein „Netz- oder Molekularsystem“. Dagegen ist die Anordnung im unteren Diagramm eher linear, wobei sie zugleich Netzwerkstrukturen in sich aufnimmt. Diese Variante bildete die von den Architekten favorisierte Version. Sie ermöglichte es, die Zielvorstellungen von sowohl Überschneidung als auch Kompaktheit zu 

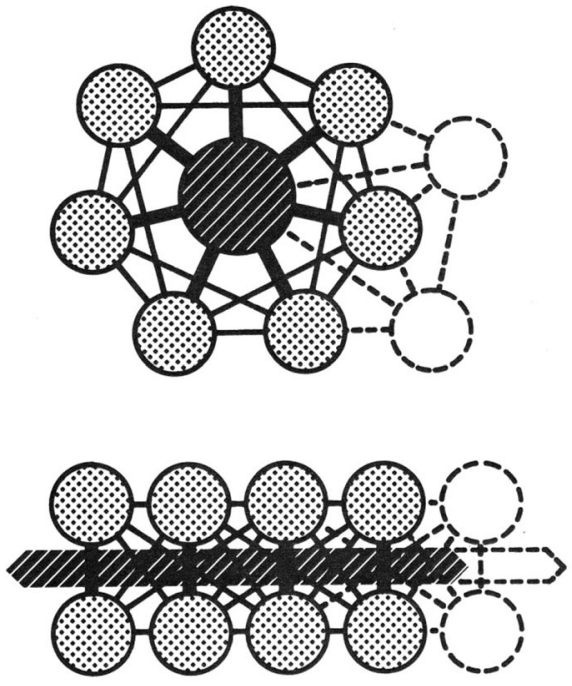

ZUORDNUNG DER FACHBEREICHE

Aus dem Reformprogramm der Universität Bielefeld ergeben sich für die Zuordnung der Fachbereiche zwei Kernbedingungen:

1) Die Forderung nach günstigen Kooperationsbedin gungen und der starke Bezug der Fakultäten auf die zentralen Einrichtungen bedingen eine enge Verflechtung. Als Bindeglied muß ein dichtes Kommunikationssystem zur Verknüpfung aller Bereiche geschaffen werden.

2) Die Forderung nach Entwicklungsmöglichkeiten, fakultät sbezogen im Kleinen (Mikroerweiterung) und auf die Universität als Ganzes bezogen im Großen (Makroerweiterung) bedingt Entwicklungs raum einerseits bei den Fakultäten für die Mikroerweiterung und andererseits Anschlußmöglichkeiten an die zentralen Einrichtungen für die Makroerweiterung.

Die Forderungen nach enger Verflechtung einerseits und Entwicklungsraum andererseits können von einem Netz - oder Molekularsystem nicht mehr erfüllt werden, da diese Systeme entweder zum Einschluß einzelner Bereiche oder aber zu einem sehr weitmaschigen Kommunikationsnetz führen.

Im Gegensatz dazu löst ein linear geordnetes System den Zuordnungskonflikt: Die vielfältigen Verbindungen sind zu einem dichten Kommunikationsband verschmolzen. In der Verlängerung der Systemachse ergeben sich direkte Anschlüsse für neue Bereiche (Makroerweiterung). Senkrecht dazu haben die beiderseitig angeordneten Fakultäten einerseits eine Randlage zur Realisierung der Mikroerweiterung und andererseits einen unmittelbaren Anschluß an das Kommunikationsband.

kombinieren und zugleich Erweiterungen zuzulassen und wurde daher als die überlegenere Version herausgestellt.

Die beiden Diagramme in Abbildung 6 wurden in der Baudokumentation von 1974 als „Systemskizze“ bezeichnet. Diese waren gegenüber der zuvor diskutierten Fassung von 1969 leicht verändert. Unter anderem deuteten nun ergänzte Pfeile Erweiterungsmöglichkeiten an. Die Gesamtkomposition der Elemente in Abbildung 6 kann verdeutlichen, wie die Variationen des Diagrammes zugleich die Baustrukturen der Universität deutbar, denkbar und imaginierbar machten. So erschien in der Baudokumentation direkt unter der "Systemskizze“ ein Foto des Wettbewerbsmodells. Auffallend ist die strukturelle Parallelität zwischen dem favorisierten Diagramm und dem Architekturmodell. So sah das Modell für das geplante Gebäude der Universität ebenfalls die Gestaltung als Band vor, das eine innere Struktur bildet und umschließt; ergänzt wurde der Hauptkörper des Universitätsgebäudes, ähnlich den Verbindungslinien des Diagramms, von querliegenden Gebäudeteilen.

Nach den Erläuterungen der Architektengruppe zum Wettbewerbsentwurf sollte diese Baustruktur die geplanten elf Fakultäten und die zentralen Einrichtungen zu einem „baulichen Kontinuum“ vereinen. Während im Sockel der Universität eine „Zone intensiver Information und Kommunikation in und an der Halle" (mit Hörsälen, Bibliotheken und Mensa) vorgesehen war, sollte darüber, mit einer Glasabdeckung „abgeschirmt“, ein „Kontinuum der ruhigen Institutsbereiche für die individuelle Arbeit" entstehen [Herzog et al. (Hg.) 1969: 20]. 


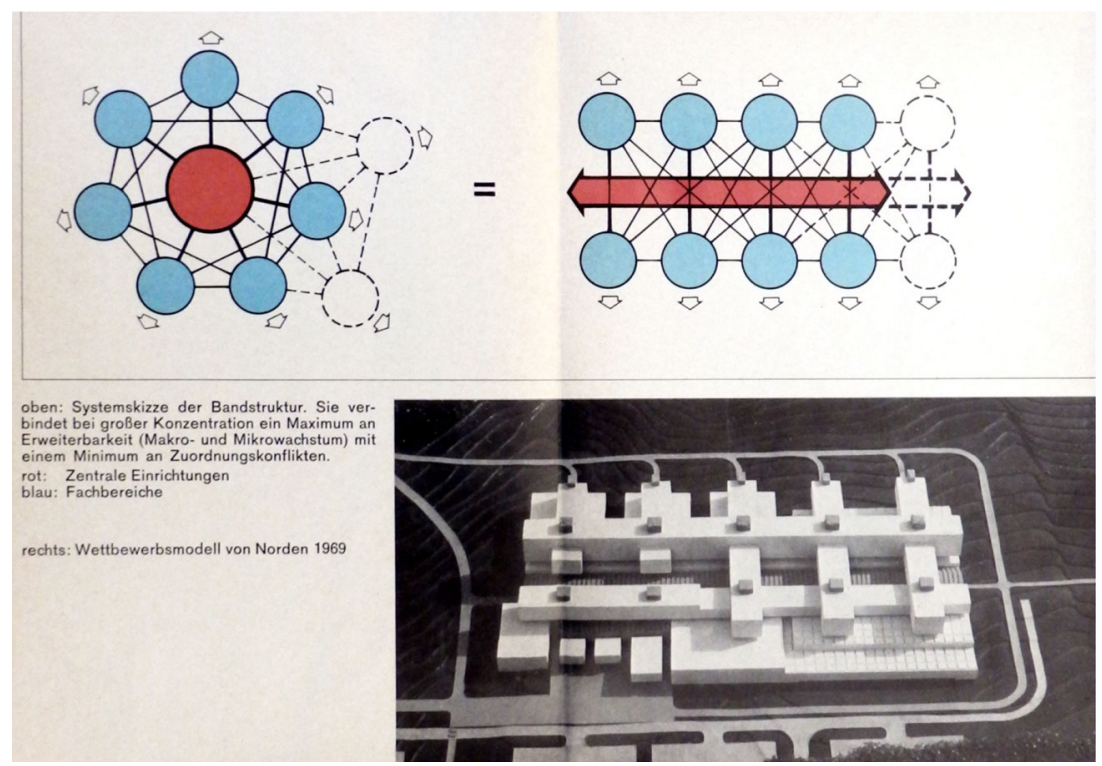

Abb. 6 Bauplanungsdokumentation Bielefeld (Universität Bielefeld (Hg.) 1974: abfotografierter Ausschnitt)

Wichtig sind an dieser Stelle die Überblendungen zwischen Systemskizze und Modell einerseits, Annahmen und Deutungen zu Kommunikation und Kooperation andererseits. Denn mit der Visualisierung von „Linearität“ war es eine Logik der Systemskizze, welche in den Erläuterungen der Architekten rhetorisch in die Figur eines „,zentrale[n] „Kommunikationsband[es]“ (ebd. 20) überführt wurde. Die bildlich angedeuteten Überschneidungen der Linien wurden als „Kreuzungspunkte[...]“ ausgelegt und zu einer „Kette von Kommunikationsschwerpunkten“ zusammengedacht. Die Bildlichkeit legte, in Verbindung mit den beigefügten Texten, somit die Rechnung nahe: (Räumliche) Einheit und Kompaktheit, in Kombination mit Schnittmengen und Netzwerken, ergibt eine Möglichkeitsbedingung von Kommunikation und Kooperation. Dadurch wurden Raumstrukturen als Ermöglichungsbedingung disziplinenübergreifender wissenschaftlicher Praxis deutbar gemacht und über das Architekturmodell für die bauliche Umsetzung empfohlen (ebd. 44).

Im Fall der Universität Bochum diente das Zuordnungsschema des Gründungsausschusses ${ }^{38}$ sogar ausdrücklich als Grundlage des Ideenwettbewerbs, der 1962 zur Entwicklung des künftigen Hochschulgebäudes ausgeschrieben wurde; dieser machte die „Zuordnung der verschiedenen Universitätsdisziplinen zueinander unter Berücksichtigung des erwähnten Zuordnungsschemas und der erwünschten engen Verflechtung“ zur Wettbewerbsaufgabe. $^{39}$ In den Berichten und Dokumentationen zum Bau der Universität Bochum finden sich mehrere Versionen des Zuordnungsschemas 


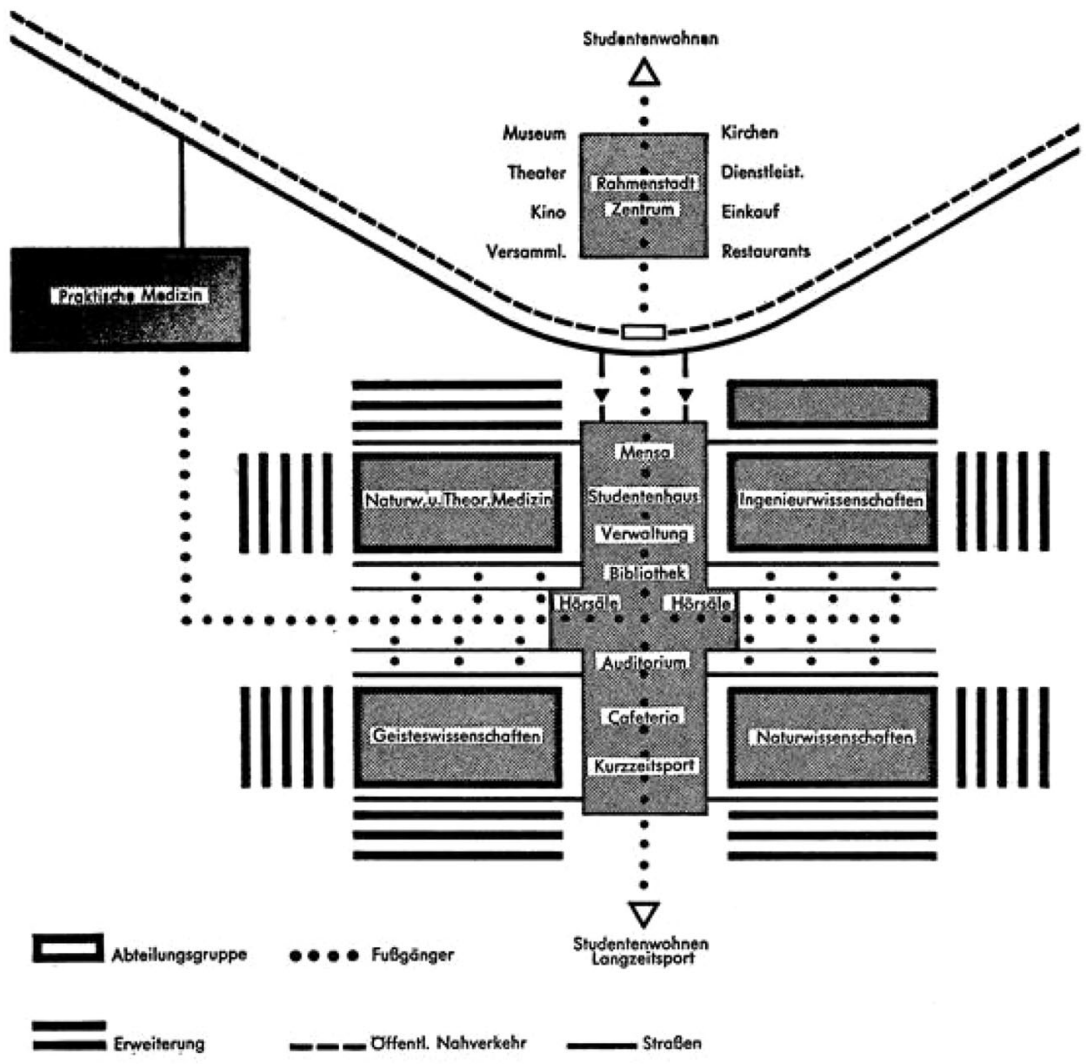

Abb. 2

Strukturschema

Abb. 7 Schema der Koordinierung Bochum (Der Minister für Landesplanung, Wohnungsbau und öffentliche Arbeiten des Landes Nordrhein-Westfalen 1965: 58)

sowie weitere Diagramme und Grafiken, welche die bauliche Form der Universität als räumliche Beziehung der Disziplinen reflektierten. ${ }^{40}$

Abbildung 7 zeigt ein "Schema der Koordinierung“ der Universität Bochum, das in der Baudokumentation von 1965 abgedruckt wurde. Dargestellt durch schwarze Rechtecke, ordneten sich in diesem Schema vier Blöcke der Naturwissenschaften und der Theoretischen Medizin, der Geisteswissenschaften, der Ingenieurwissenschaften und der Naturwissenschaften um einen Zentralbereich mit Einrichtungen wie Audimax, Cafeteria und Bibliothek an. Leicht ausgegliedert links oben referierte ein schwarzes Rechteck auf die zunächst angedachte Praktische Medizin. Im oberen Teil des Schemas, jenseits geschwungener Linien für Nahverkehrslinien und Erschließungsstraße, erschien ein schwarzes Quadrat mit der Bezeichnung „Rahmenstadt 


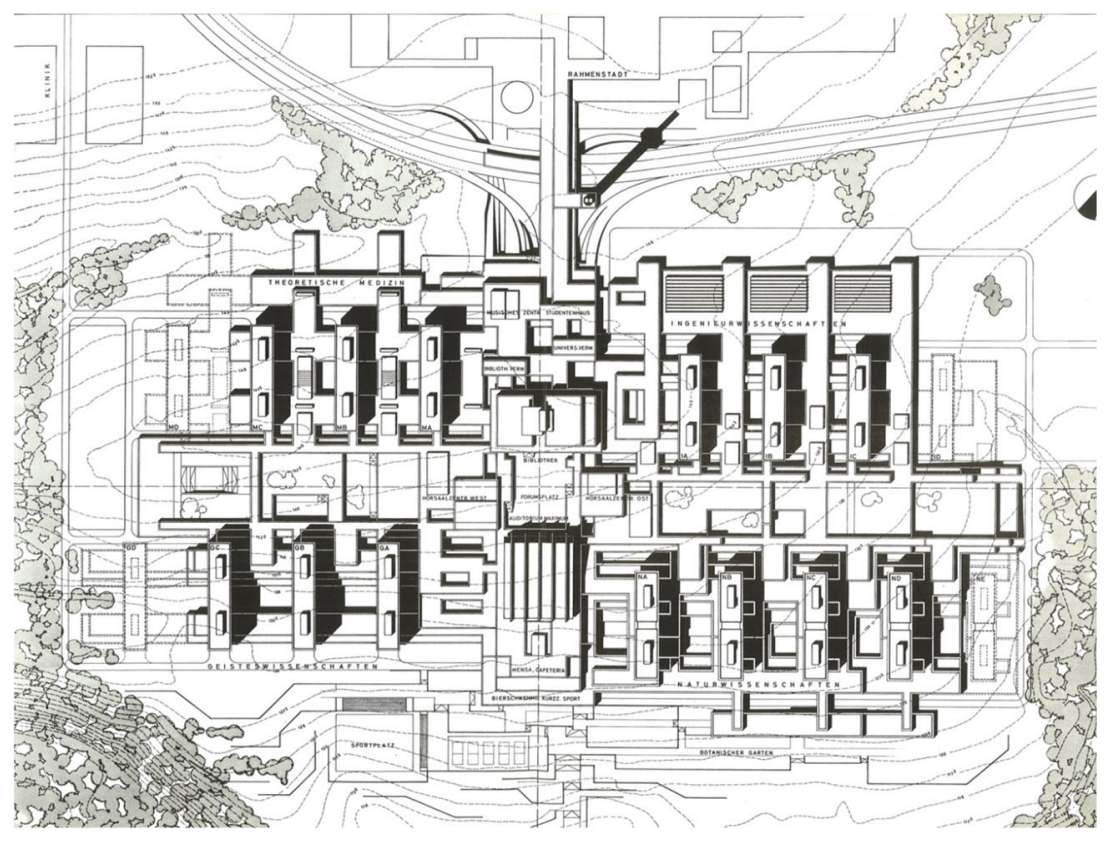

Abb. 8 Bauplan Bochum (Der Minister für Landesplanung, Wohnungsbau und öffentliche Arbeiten des Landes Nordrhein-Westfalen (Hg.) 1965, Scan des Universitätsarchivs Bochum)

Zentrum“. Punkte legten geplante Fußwegbeziehungen nahe, Linien angedachte Erweiterungsmöglichkeiten.

Abbildung 8 zeigt im Vergleich dazu den Bauplan der Universität. Man mag darüber spekulieren, inwiefern das oben dargestellte Schema der Universität Bochum wiederum Formen aufgriff, die letztlich aus dem Zusammenrücken von Text zu komplexeren Formen der Schriftbildlichkeit entstanden waren (vgl. dazu Abb. 1 und 2). In jedem Fall verdeutlichen die hier diskutierten Beispiele, wie visuelle Entwürfe zur Auseinandersetzung um interdisziplinäre Wissenschaft beitragen konnten. So führten Schemata und Diagramme Diskurse der disziplinären Zersplitterung, der Fragmentierung, der verlorengegangenen „Einheit der Wissenschaft“ und der notwendigen „Grenzüberschreitung“ weiter zu Visionen von Universität und Wissenschaft etwa als „Netzwerk“, als Überkreuzung von Linien; diese Visionen wurden in der Planung von Hochschulgebäuden architektonisch zu Raumkonstitutionen auf der Basis räumlicher Nähe fortentwickelt. Diagramme, Pläne und Kartierungen konstituierten Annahmen über Wissenschaftlichkeit auch als visuelle Raumrelation. Schließlich trugen Bilder und Modelle dazu bei, Konzeptionen disziplinenübergreifender Wissenschaft über die Materialität ihrer gebauten Umwelt plausibel $\mathrm{zu}$ machen. Fächergrenzen $\mathrm{zu}$ überschreiten und Verständigung an einem Ort anzuregen, konnte dadurch zu einer Zielvorstellung werden, welche sich auch der visuellen Kultur der 
Hochschulgründungen der 1960er Jahre in ihrer Verwobenheit von Raumund Wissenschaftskonzeptionen verdankt.

\section{Fazit: „Interdisziplinarität” als Zeitgeschichte}

Reflexionen interdisziplinärer Wissenschaft kursierten also in verschiedenen Wissensfeldern und Artikulationsformen. Beginnend mit wissenschaftlichen Texten der 1950er und 1960er Jahre, führte dieser Beitrag über wissenschaftspolitische und wissenschaftsorganisatorische Texte im Umfeld der Hochschulneugründungsdebatten hin zu visuellen Auseinandersetzungen um disziplinenübergreifende Kooperation und Kommunikation. Es liegt damit nur nahe, zum Schluss dieser Betrachtung noch einmal zur Sprache der Wissenschaft zurückzukehren. Denn einerseits Teil der wissenschaftspolitischen Debatte, bildete interdisziplinäre Wissenschaft andererseits auch in wissenschaftlichen Texten der 1960er Jahre einen Anlass zur wissenschaftlichen Selbstreflexion.

So hoben wissenschaftliche Publikationen interdisziplinäres Vorgehen als „fruchtbar" hervor. ${ }^{41}$ Disziplinenübergreifende Wissenschaft sollte eine Orientierung an ,internationalen“ und vornehmlich US-amerikanischen Vorbildern ermöglichen; sie wurde als „notwendig“"42 und „erforderlich“43 ausgezeichnet und konnte sich mit Zuschreibungen von „Kühnheit“ oder der Aussicht auf „Innovationen des Denkens“ (Edding 1965: 1) verbinden. Dieser Erwartungshaltung folgend, stellten manche Veröffentlichungen dieses Jahrzehnts interdisziplinäre Vorgehensweisen als nachgerade imperativ heraus („Realistische Entwicklungsforschung kann nur interdisziplinär sein“). ${ }^{44}$ Eine solche Alternativlosigkeit interdisziplinärer Forschung wurde auch durch die Begrifflichkeiten betont, mit denen im Gegensatz dazu rein disziplinär orientierte Forschung bedacht werden konnte. Die Kehrseiten disziplinärer Spezialisierung hervorhebend, akzentuierten Wissenschaftler etwa die „Notwendigkeit der Überwindung disziplinärer Schranken“ (Krippendorff 1965: 194), widersagten den „Scheuklappen einer streng abgegrenzten Einzeldisziplin“ oder wollten einem „Provinzialismus disziplinärer, nationaler, methodologischer Begrenzungen" entgehen (Pfeffer 1963: 36-37).

Trotz dieses Nachdrucks bedingten Forderungen nach interdisziplinärer Wissenschaft keine eindeutigen Anweisungen, was diese ausmache und wie diese umzusetzen sei. So charakterisierte Reinhart Koselleck Interdisziplinarität in den 1970er Jahren als ein „leeres Schlagwort" beziehungsweise - ins Freundliche abstrahiert - als „ein Programm von hoher Formalität“ ${ }^{45}$ Konzeptuell blieb der Aggregatzustand interdisziplinärer Wissenschaft insofern eher flüssig. ${ }^{46}$ Gemeinsam war den in diesem Beitrag betrachteten Auseinandersetzungen um disziplinenübergreifende Kooperation allerdings, dass sie 
bestimmte Modi des Erkennens anderen vorzogen. Wissenschaft erschien in ihnen als ein gemeinschaftliches Unternehmen, dessen Protagonisten sich austauschen und über ihre Disziplinen hinweg das Gespräch suchen sollten. Auch als Folge informellen Zusammentreffens gedacht oder zumindest als mögliches Ergebnis nicht völlig planbarer Kontakte zwischen Wissenschaftlern imaginiert, richteten sich solche Gespräche nach dem Willen ihrer Verfechter nicht lediglich darauf, einzelnen wissenschaftlichen Desideraten nachzugehen. Dem Austausch zwischen den Disziplinen wurde vielmehr teils auch das Potenzial zugeschrieben, ein Auseinanderrücken wissenschaftlicher Teilbereiche zumindest situativ aufzufangen und der Annahme Nachdruck zu verleihen, dass Wissenschaft mehr bedeuten müsse als ein bloßes Nebeneinander von Einzeldisziplinen.

Das Zusammenspiel von normativer Erwartung und relativer Bedeutungsoffenheit, das eingangs für die Gegenwartsdebatten um Interdisziplinarität bemerkt worden ist, charakterisierte die Auseinandersetzung um interdisziplinäre Forschung und Lehre in der Bundesrepublik also bereits in den 1960er und 1970er Jahren. Um dies besser zu verstehen, mag es hilfreich sein, interdisziplinärer Wissenschaft nicht als einem Ansatz zu begegnen, der überzeitlich definierbar wäre. Gewinnbringender erscheint es, Interdisziplinarität als eine historische Denkform des Erkennens zu beschreiben, welche die Zirkulation politischer, sozialer und epistemologischer Reflexionen durch unterschiedliche Wissensfelder ermöglichte und mittels derer Modi des Erkennens auf zeitspezifische Anforderungen von Wissenschaft bezogen werden konnten. Auseinandersetzungen um Interdisziplinarität erlaubten es auf diese Weise Wissenschaftlern wie Wissenschaftsorganisatoren gleichermaßen, jeweils als drängend wahrgenommene Probleme im Nachdenken über den Zusammenhang der Wissenschaft aufzugreifen und diesen in Reflexionen etwa über das Verhältnis von Teil und Ganzem der Wissenschaft zu begegnen.

Gerade wegen ihres eher unspezifischen Charakters konnten die konkreten Themen und Fragen, die in Debatten um interdisziplinäre Wissenschaft aufgegriffen wurden, variiert werden und dadurch die Attraktivität der Denkform über veränderte Kontexte hinweg erhalten. In den 1960er Jahren waren die Öffnung der bundesdeutschen Wissenschaft gegenüber internationalen Wissenschaftsentwicklungen sowie Herausforderungen durch den quantitativen Ausbau der Universitäten die zentralen Themen, die Auseinandersetzungen um Interdisziplinarität untergründig konturierten. Nicht zuletzt im Zuge der Politisierungswelle im Gefolge der Studentenbewegung und der entstehenden neuen sozialen Bewegungen gewann seit den 1970er Jahren zudem das Argumentationsmuster an Gewicht, durch interdisziplinäre Wissenschaft die Relevanz der Forschung zu steigern und die Probleme der Gesellschaft - und nicht die der Disziplinen - angehen zu wollen. ${ }^{47}$ Insbesondere mit der Betonung von Umwelt- und Friedensfragen in den 1970er und 
1980er Jahren versprach interdisziplinäre Forschung geeignet zu sein, ,politisch und gesellschaftlich aktuelle Kernprobleme [aufzunehmen, S. Sch.], die von außen an das ,System Wissenschaft' herangetragen werden“ (Voßkamp 1984: 460). „Interdisziplinäre“ Forschung erschien nun „mit der Lebenswelt häufig unmittelbarer verknüpft als disziplinäre Forschung“ (ebd.) und forderte dadurch dazu auf, sich den „Forschungs- und Planungsaufgaben“ zuzuwenden, „von denen unser Überleben abhängt“ (von Hentig 1971: 860 f.).

Insofern der Rekurs auf Interdisziplinarität aber nun auch dazu diente, angestrebte Eigenarten von Wissenschaft zu formulieren und sich über ihre Ziele zu verständigen, war die Enttäuschung dieser Visionen durch die Praxis bereits angelegt. Neben der Gefahr eines "gehobenen Dilettantismus“ oder einem „Zwang zur Pseudo-Universalität" (Nail 1975: 32, Zitat aus der rezensierten Schrift) beklagten Wissenschaftler bald die „Schwierigkeiten interdisziplinärer Kommunikation, die bei unterschiedlichem Niveau der methodologischen und theoretischen Grundlagen kaum überbrückbar sind“ (Blankenburg et al. 1972: 600); sie resümierten, „daß die oft geforderte Interdisziplinarität vielfach schwer durchsetzbar ist" (Kaiser 1975: 205), oder gaben zu Protokoll, „daß nicht nur die Hoffnungen auf Inter-Disziplinarität, sondern selbst die auf unkonventionelle Kooperationen [...] rasch enttäuscht wurden“ (Die Jahrbücher 1978). Ein wissenschaftlicher Text vermerkte zum Ende der 1970er Jahre gar, es seien die „Verständigungsschwierigkeiten dermaßen groß, daß von Interdisziplinarität kaum mehr ohne Resignation oder Ironie die Rede ist“; das Substantiv sei „offenbar ein Wort, das nur noch bei Anträgen auf Forschungsfinanzierung sich auszuzahlen scheint" (Heyen 1978: 509).

Der fortwährenden Präsenz von Forderungen nach interdisziplinärer Wissenschaft haben Gegenreden dieser Art dennoch kaum geschadet. In der Wissenschaftssprache der Bundesrepublik, in der Forschungsförderung ${ }^{48}$ oder in Versuchen der Studienreform (Schlager 2014; Holtorf 2014) hat die Propagierung disziplinenübergreifender Wissenschaft ihren festen Platz behalten. Gegenwartsvariationen der in diesem Beitrag verfolgten Debatten manifestieren sich zudem in Forschungsverbünden und im Umfeld der älteren wie im Zuge der Exzellenzinitiative neugegründeten - Institutes for Advanced Studies. ${ }^{49}$ Und auch in der Gegenwart dient der Bezug auf Interdisziplinarität der Reflexion auf übergeordnete Ziele und Verpflichtungen der Wissenschaft. Nur so zumindest ist es zu verstehen, wenn die Zeitschrift Nature ihr aktuelles Special Issue zum Thema unter der Überschrift „How to solve the world's biggest problems“ präsentiert. Dieses begleitet eine Comiczeichnung von Wissenschaftler/innen, die in einem Outfit irgendwo zwischen Astronaut, Superman und Mangaheld mit geballten Fäusten in den Himmel (natur)wissenschaftlicher Erkenntnis entfliegen (Trippe 2015). 
Damit scheint es, als ob wir in der Geschichte der Interdisziplinarität jenen Punkt noch nicht erreicht haben, den Hans-Jörg Rheinberger als „Wasserscheide" zwischen der Geschichte und der Zeitgeschichte der Wissenschaft beschrieben hat und der nicht in Jahren zu messen sei, sondern im „kognitiven Horizont des Historikers selbst" seine Entstehung finde. Dieser entstehe dort, „wo die verspürte Notwendigkeit, sich zu distanzieren, übergeht in die Notwendigkeit, sich einzufühlen in eine Welt, die nicht mehr die unsere ist" (Rheinberger 2006: 188). Das bloße Faktum ihrer Geschichtlichkeit wird uns dabei zwar noch keinen Maßstab an die Hand geben, mit dem gegenwärtige Debatten um interdisziplinäre Wissenschaft bewertet werden könnten. Es legt aber zumindest nahe, dass es uns hin und wieder guttun könnte, unsere Aufmerksamkeit auf die Sprache und die Bilder zu richten, in denen wir uns heute über das Überschreiten von Fächergrenzen verständigen. Ihren eigenen Regeln folgend, werden diese uns kaum einen Spiegel vorhalten, aus dem wir in direkter Weise Aufschlüsse über unsere eigene wissenschaftliche Praxis erhalten. Vielleicht jedoch erlauben sie uns einen kurzen Moment der Distanzierung, in dem wir die Historizität unserer Vorstellungen (inter)disziplinärer Kommunikation und Kooperation sei es als Last, sei es als Befreiung erleben. Eine weitere Historisierung interdisziplinärer Wissenschaft kann dadurch möglich werden.

\section{Danksagungen}

Das Thema interdisziplinärer Wissenschaft hat auch meine eigenen wissenschaftlichen Wege begleitet. Für Diskussionen danke ich daher meinen Kolleginnen und Kollegen aus dem Graduiertenkolleg „Topologie der Technik“ (TU Darmstadt), der Forschergruppe „Werkzeuge des Entwerfens“ (IKKM Weimar), dem a.r.t.e.s. Research Lab an der Universität zu Köln sowie der Klasse 5 der a.r.t.e.s. Graduate School for the Humanities Cologne. Viele Anregungen habe ich zudem von der Jahrestagung der Gesellschaft für Medienwissenschaft 2013 („Medien der Wissenschaften“) erhalten, auf der ich eine erste Version dieses Beitrags vorstellen durfte. Für wichtige Hinweise und Verbesserungsvorschläge danke ich zudem David Sittler, Till van Rahden, Heike Weber sowie den beiden anonymen Gutachtern. Der Beitrag wurde fertiggestellt während eines Forschungsaufenthaltes am Institute for Advanced Studies in the Humanities (IASH) Edinburgh, ermöglicht durch das EURIASProgramm sowie die European Commission Marie-Sklodowska-Curie Actions - COFUND Programme - FP7. 


\section{Anmerkungen}

1 Zur Nützlichkeit begriffsgeschichtlicher Ansätze für die Wissenschaftsgeschichte vgl. Schauz 2014; 2015 (mit der Anregung, sich stärker der Analyse „metawissenschaftlicher Leitbegriffe“ zuzuwenden); Eggers \& Rothe 2009, 7-14; Müller \& Schmieder 2008. Aus der Perspektive der Zeitgeschichte, insbesondere mit Blick auf mögliche Spezifika einer Begriffsgeschichte des 20. Jahrhunderts, Geulen 2010; Hoffmann \& Kollmeier (Hg.) 2012.

2 Derzeit scheint die "Interdisziplinarität der Begriffsgeschichte“ eher ein Thema der Forschung zu sein als die Begriffsgeschichte der Interdisziplinarität. Dies ist umso mehr verwunderlich, als die Begriffsgeschichte der Interdisziplinarität selber auch Teil der Geschichte der Begriffsgeschichte ist. Zu Ersterem vgl. etwa Veit-Brause 2000; Schauz 2014: 59; Müller \& Schmieder 2008: XI, XVII; oder die Publikationsreihe „Forum Interdisziplinäre Begriffsgeschichte“.

3 Zur Begriffsgeschichte der Interdisziplinarität vgl. zunächst Holzhey 1976; Balsiger 2005: 157-173; Historisches Wörterbuch interdisziplinärer Begriffe 2015: Stichwort „Interdisziplinarität“. Eine genauere Diskussion erfolgt im folgenden Teilkapitel.

4 Als Verwendungsnachweis des Substantivs in wissenschaftlichen Texten vgl. etwa Zweite Loccumer Reform-Tagung 1970: 20 („An Stelle der ,intrapersonalen` Kooperation, bei der jeder Partner unkritisch von der anderen wissenschaftlichen Disziplin abhängt, wollte er [Erich Küchenhoff, S. Sch.] die ,intrapersonale Interdisziplinarität' setzen“); von Hentig 1971: 860 f. („INTERDISZIPLINARITÄT - dieses Wort erinnert daran, daß die heutige Gliederung der Universität dem ausgehenden 18. Jahrhundert entstammt und sich gegen die Erfüllung der realen Aufgaben sperrt, die das Leben heute der Wissenschaft stellt. [...] Alle Interdisziplinarität ist bisher in die Disziplinarität gemündet und hat die Kooperations- und Kommunikationsnot in der Wissenschaft nur vermehrt"); Rehbinder \& Schelsky (Hg.) 1972: 599 („Diskussion zur Methode der Interdisziplinarität“); Schelsky 1972: 603 (vielleicht entstehe einmal eine „Theorie der Interdisziplinarität“; ratsamer erscheine es dagegen, „die Erfahrungen interdisziplinärer Zusammenarbeit an konkreten Themen und von sehr verschiedenen Fächern vorangehen zu lassen“); Mühlenberg 1972: 5, 13 („Erläuterungen zur Definition von Interdisziplinarität“).

5 Zusammenfassend Thompson Klein 1990: 19-39; Hübenthal 1991: 4-6; Hilgendorf 2010: 918-919; Kocka 2008. Speziell zu den Klagen über einen „Verlust des Ganzheits- und Zusammenhangsbewußtseins" in der unmittelbaren Nachkriegszeit sowie korrespondierenden Konzeptionen eines „Studium Generale“ vgl. Papenkort 1998; Zum Geleit 1947.

6 Vgl. etwa Cohen-Cole 2014: 65-103; Thompson Klein 1990: 19-39; Frank 1988; Sills 1986.

7 Vgl. Frodeman et al. 2010; Jungert 2010; Weingart 1995; Weingart \& Stehr 1999; Balsiger 2005; Hübenthal 1991; Mittelstraß 2003.

8 Vgl. etwa Hoppe-Sailer et al. 2015: 9-11; Löning et al. 2011; Paulus 2010; Muthesius 2000; Sprenger \& Weingart 1994. Einen ersten Ansatz, den Stellenwert der Forschungsförderung bei der Propagierung von Interdisziplinarität herauszuarbeiten, macht zudem Weingart 2002: 178-190 in einem Jubiläumsband der Volkswagenstiftung.

9 Vgl. dazu einstweilen Balsiger 2005: 170-171; als Beispiel stärker politisierter Sprachverwendung siehe etwa Albuschewsky 1968 („Interdisziplinäre Forschung - das scheint mir heute der einzig mögliche Weg zu sein, um den von Walter Ulbricht [...] geforderten Welthöchststand in Ausbildung und Forschung in kurzer Zeit zu erreichen“); Kröber 1983.

10 Suche in Arts \& Sciences 1-5, 7-14; Biological Sciences; Health \& General Sciences, Suchlauf November 2015.

11 Wie etwa „cooperative research“, „interrelation“, „mutual interdependence“, „interprenetration“, „intercommunication“, „cross-relationships“, „interfiliations“, „interactions“ (Frank 1988: 93-95).

12 Als Belegstellen aus den 1950er Jahren ohne direkten Bezug zur US-Wissenschaft siehe so Engelhardt 1957: 181; Stachowiak 1957: 403; Kyle 1959: 213, 219; Krüsselberg 1959: 130; Bühler 1959: $11 \mathrm{f}$. 
13 Paul 1955: 380; Paul 1957; Bergsträsser 1956; Bahrdt et al. 1960.

14 Gäfgen 1961: 2; Stammer 1961: 100; Fischer 1962: 530; Stöhr 1962: 22-23; Fischer 1963: 395, 400-401; Albert 1963; Czempiel 1965: 280; Krippendorff 1965: 193-196.

15 So (in chronologischer Folge): Schlochauer 1958: 222 (Besprechung von Georg Schwarzenberger 1956, „The Province of the Doctrine of International Law") Schwarzenberger lehrte als deutscher Immigrant in London; Kade 1960 (in seiner Rezension von G. T. Guilbaud, What is Cybernetics, London u.a. 1959); Albert 1962 (im Verweis auf Robert A. Dahl, Social Science Research in Business, New York 1959); Ambros 1962: 559 (in seiner Rezension des Werks des deutschen Immigranten William K. Kapp, Toward a Science of Man in Society, The Hague 1961. Kapp arbeitete an der Columbia University); Blanke 1963 (Rezension von Sigmund Skard, The American Myth and the European Mind, Philadelphia 1961); Herzog 1964 (Rezension von Soziologie der Demokratie, dt. Übersetzung von Seymour Martin Lipset, The Political Man, New York 1960); Edding 1965: 1, 4 (Rezension von Fritz Machlup, The Production and Distribution of Knowledge in the United States, Princeton/New Jersey 1962); Robinsohn 1965: 234 (Rezension von George Bereday, Comparative Methods in Education, New York 1964); Wander 1965 (Rezension zu National Bureau of Economic Research (Hg.), Demographic and Economic Change in Developed Countries, Princeton 1960). Auf eine französische Publikation Bezug nimmt Kaletsch et al. 1964: 743.

16 Vgl. etwa Kandel 1959; Stöhr 1962: 22, 23; Lauwerys \& Robinsohn 1963: 131; Nadler 1964: 23.

17 Kade 1965 (über Mesarovic).

18 Vgl. etwa Winsemius 1960: 134; Robinsohn 1965: 234.

19 Zur Wissenschafts- und Universitätsgeschichte nach 1945 vgl. zusammenfassend Klein et al. 2014. Speziell zur Hochschulgeschichte der 1960er bis 1980er Jahre siehe Rohstock 2010; Paulus 2010: 477-524; Kenkmann 2000; Jessen \& John 2005.

20 Bereits 1962 erschien die Forderung nach „interdisziplinären“ Lehrformaten so im Gutachten des VDS „Studenten und die neue Universität“. Vgl. Verband Deutscher Studentenschaften 1962: 57-58, mit der Forderung: „Im Studium soll der Student die Möglichkeiten der Kooperation erfahren. [...] Kommunikation mit anderen Disziplinen geschieht durch interdisziplinäre Lehrveranstaltungen und durch die Beteiligung des Studenten an interdisziplinärem Arbeiten." Als ein Vorlesungsformat wird u.a. vorgeschlagen: „das interdisziplinäre Kolleg, in dem Grenzprobleme und fachübergreifende Fragen aus der Forschung und der dort geübten Kooperation heraus von mehreren Hochschullehrern behandelt und vermittelt werden." Auch „Nachdiplomstudien“ sollten nach dem Willen des VDS unter anderem "der Teilnahme an interdisziplinärer Arbeit" dienen. Vgl. ebenso die Ausführungen zur Gründung „Interdisziplinärer Institute“ ebd. 29-31. Siehe auch Jüchter 1963, sowie zum Hintergrund Rohwedder 2011.

21 Zur „Einheit” der Wissenschaft und ihrem drohenden Verlust bzw. ihrer Aufhebung vgl. auch Galison 1996: 1-8.

22 Empfehlungen zur Gründung der Universität Bochum [1962] 1968: Nr. 388-Nr. 571, Zitat Nr. 392.

23 Die Universität Konstanz [1965] 1968: Nr. 1432-1442 (Abschnitt zu „Formen der Kooperation"), Zitat Nr. 1432.

24 Schelsky [1965] 1966b: 35-70; zum Hintergrund Söllner 2013.

25 Vgl. etwa Schelsky [1965] 1966b; Gründungsausschuß [1966] 1968: Nr. 369, 376, 378, 386.

26 So erklärte Schelsky, das Zentrum für interdisziplinäre Forschung sei „der Versuch, die besonders in den USA bewährte Einrichtung der, Centers for Advanced Studies' auf das deutsche Wissenschafts- und Hochschulsystem zu übertragen und dafür fruchtbar zu machen. Wir sehen in dieser Institution den einzigen aussichtsreichen Versuch, die an den Universitäten längst nur noch formal vorhandene ,Einheit der Wissenschaften“ noch einmal unter den Bedingungen der modernen Wissenschaftsentwicklung zu verwirklichen; es ist daher der ,Akademie-Gedanke', der unserer Zeit entspricht" (Schelsky [1965] 1966b: 42-44). Zu diesen Einrichtungen vgl. auch Converse 2001, zum ZiF Sprenger \& Weingart 1994.

27 Vgl. so (in chronologischer Folge): Aus der Entwicklungsländer-Forschung 1960: 162 („Arbeitsgemeinschaft ,Entwicklungsländer' an der Universität Freiburg“); Burrichter 1963: 371 (Forschungsvorhaben „Möglichkeiten einer interdisziplinären Erforschung 
industrialisierter Arbeitsvollzüge“, Soziologisches Seminar der Universität Göttingen); Bornemann \& Nave-Herz 1964: 283 (zum 1964 gegründeten „Institut für Bildungsforschung" in der Max-Planck-Gesellschaft); Danckwortt 1964 (zum 1959 geschaffenen Seminarzentrum der Deutschen Stiftung für Entwicklungsländer); Pfeifer 1964: 139 (zum von der DFG unterstützten „Mexiko-Projekt“); Sonnenhol 1965: 25 (zum Institut für Entwicklungspolitik in Berlin, das 1965 eröffnet wurde); Max-Planck-Institut für biologische Kybernetik 1968 (zur Gründung dieses Instituts in Tübingen).

28 Die Suche nach dem Adjektiv ,interdisziplinär" zeitigte so im (1949 einsetzenden) digitalen Archiv der Frankfurter Allgemeinen Zeitung erste Ergebnisse für die frühen 1960er Jahre, mit stark zunehmender Tendenz zum Ende des Jahrzehnts (1961: 1 Treffer, 1963: 2 Treffer, 1964: 2 Treffer, 1965: 6 Treffer, 1966: 11 Treffer, 1967: 18 Treffer, 1968: 32 Treffer, 1969: 49 Treffer). Zum Suchwort „Interdisziplinarität“ erschien der erste Treffer 1971. Suchlauf am 04.02.2015.

29 Zu Bildern in der Wissenschaft und Technik siehe Mößner 2012: 9-14; Gall 2009; Norton Wise 2006; Heßler 2005.

30 Zum Entwurf vgl. Siegert 2009: 19-23; Wittmann 2012: 136-138; Gethmann \& Hauser 2009: 9-11.

31 Die Ausführungen des Gründungsausschusses finden sich in: Empfehlungen zur Gründung der Universität Bochum, in: Neuhaus (Hg.), Nr. 388-Nr. 571. Aufgrund der einfacheren Zugänglichkeit zitiere ich nach Neuhaus. Die Abbildungen sind aber hier entnommen aus: Empfehlungen zum Aufbau der Universität Bochum. Denkschrift des Gründungsausschusses, Bochum (Dezember) 1962: 11. Gegenüber der Version bei Neuhaus (Hg.): Nr. 400, ergeben sich durch einen Seitenumbruch leichte Abweichungen.

32 Vgl. zur Hochschularchitektur Muthesius 2000; zu räumlich-architektonischen Interdisziplinaritätsdebatten Heßler 2007: 157-158; 305-307.

33 Zur Debatte um die Räume und Orte des Wissens und die Architekturen der Wissenschaft zusammenfassend Henke \& Gieryn 2007.

34 Ausschreibung des Ideenwettbewerbs. Die Baukonzeption, Düsseldorf, 04.07.1962, Akte beim Staatshochbauamt, Nr. 022, S. 4 f., zitiert nach von Cube 1992: 29. Zum Ideenwettbewerb vgl. auch Apfelbaum \& Schmitz 2015: 60.

$35 \mathrm{Zu}$ den im Folgenden vertieften Beispielen Bochum und Bielefeld vgl. Hoppe-Sailer et al. 2015: 9-11; von Cube 1992: 145-191; stark wertend Schmidtke 2007; Braungart 2009; Weisner 1994; Löning et al. 2011.

36 Vgl. neben dem Folgenden auch: Empfehlungen zur Gründung der Universität Bochum (1962), Nr. 396; Über die Gründung einer Universität zu Bremen (1960), Nr. 662-663; Tonutti 1977: 12.

37 Universität Bielefeld (Hg.) 1974: abfotografierter Ausschnitt.

38 Vgl. dazu die Ausführungen bei Abb. 1 und 2.

39 Zitat Städtebaulicher Ideenwettbewerb 1963: 276-277. Dazu auch Hallauer 1990: 216223, zum Zuordnungsschema ebd. 212 f.; von Cube 1992: 25-30; Apfelbaum \& Schmitz 2015: 60.

40 Siehe etwa Stüer [1963] 1965: 25; Franken 1965; Bittel 1972; Hallauer 1990: 211.

41 Vgl. etwa Pfeffer 1963: 33; Kade 1962b: 276 („gegenseitige Befruchtung einzelner Disziplinen“); Stöhr 1962: 22 (,interdisziplinäre Richtung mit systematisch herbeigeführter Befruchtung durch Nachbardisziplinen").

42 Stachowiak 1957: 403 („die dringend notwendige interdisziplinäre Verständigung“); Kade 1962b: 276 („Die bisherigen Ergebnisse, die sich hieraus für die Sozialwissenschaften ergaben, beweisen zweifellos die Zweckmäßigkeit der interdisziplinären Zusammenarbeit; ihre Notwendigkeit sollte nicht mehr zur Debatte stehen: The proof of the pudding is in the eating!").

43 Kade 1962a: 477 („Gerade für die Entscheidungstheorie ist eine interdisziplinäre Zusammenarbeit erforderlich, weil hier besonders deutlich wird, daß ein Problem durch die Menge möglicher Fragestellungen, nicht durch die historisch gewachsene Arbeitsteilung der Wissenschaften begrenzt ist"); Frenckner 1965: 125 („Im Vergleich zu der gegenwärtigen Organisation der Forschung insbesondere in Europa würden interdisziplinäre Forschungsgruppen und internationale Zusammenarbeit bei der Untersuchung dieser Elemente als Zellen des Unternehmensprozesses zu einer spürbaren Verbesserung führen. Die gleiche Art der interdisziplinären und vielleicht auch internationalen 
Forschung scheint für das Verständnis der verschiedenen unternehmerischen Informations-, Steuerungs- und Entscheidungsprozesse erforderlich").

44 Den Menschen mobilisieren 1964: 5, Referat Richardt Behrendt. Vgl. auch Pfeffer 1963 („Eine fruchtbare soziologische Orientforschung kann nur interdisziplinär vorgehen“); Lauwerys \& Robinsohn 1963: 131 („Es wird weitgehend zugegeben, daß Vergleichende Erziehungswissenschaft als eine synoptische Form der Untersuchung auf interdisziplinäre Forschung und Zusammenarbeit angewiesen ist"); Nadler 1964: 23 („Darüber hinaus sollte das I[ndustrial] E[ngineering, S. Sch.] insbesondere Vorkämpfer der interdisziplinären Forschung sein“). Vgl. auch Flohr 1965: 73 (mit der Annahme, „daß eine gründliche wissenschaftliche Beschäftigung mit allen einschlägigen Fragen interdisziplinäre Zusammenarbeit verlangt").

45 Koselleck [1978] 2010: 52; vgl. auch Vowinckel 2014.

46 Vgl. zur These einer Verflüssigung von Begriffen im 20. Jahrhundert Geulen 2010: Abschn. 9-10; Weiterführung durch Steinmetz 2012: 98-99.

47 Diese Tendenz zeigte sich auch aus einer internationalen Perspektive, wenn eine Konferenz der OECD 1970 Interdisziplinarität als einen Ansatz herausstellte, die Relevanz der Wissenschaft zu steigern. 1982 brachte eine Nachfolgekonferenz dies auf den Slogan: "Communities have problems, universities departments“ (CERI (Hg.) 1982. The University and the Community. Zitiert nach Arnold 2010: 7).

48 Vgl. etwa Sondermann et al. 2008: 84-92 zur Exzellenzinitiative, oder die aktuellen Förderlinien der Volkswagenstiftung.

49 Etwa wenn diese ostentativ eine bestimmte Form wissenschaftsfördernder Sozialität auf der Basis von Informalität begrüßen und als eine Inspiration fächerübergreifender Kontakte hervorheben. Siehe dazu etwa Röttger-Rössler 2005; Giuliani 2015; Frick 2009; Freiräume 2009; Interdisziplinarität 2013; Langewiesche 2005.

\section{Literatur}

Abbott, Andrew 2001. Chaos of Disciplines. Chicago: The University of Chicago Press.

Albert, Hans 1962. Eine Theorie des Marktverhaltens großer Unternehmungen. Jahrbücher für Nationalökonomie und Statistik (174): 191-198.

Albert, Hans 1963. Rezension zu Sidney Siegel, L. E. Fouraker, Bargaining and Group Decision Making, New York u.a. 1960. Jahrbücher für Nationalökonomie und Statistik (175): 151-153.

Albuschewsky, Monika 1968. „Im Sinne des Marxschen Menschenbildes“. Neues Deutschland (05.05).

Ambros, Dankmar 1962. Rezension zu William K. Kapp, Toward a Science of Man in Society. Zeitschrift für die gesamte Staatswissenschaft (118): 558-561.

Anregungen des Wissenschaftsrates zur Gestalt neuer Hochschulen. Verabschiedet von der Vollversammlung des Wissenschaftsrates am 10. Februar und 26. Mai 1962. In: Neuhaus (Hg.): Nr. 1-Nr. 122.

Apfelbaum, Alexandra und Frank Schmitz 2015. Universitas durch Dichte. Der Ideenwettbewerb zur Ruhr-Universität 1962/63. In: Hoppe-Sailer et al. (Hg.): 59-77.

Arnold, Markus 2010. Academic Gatekeeping. Grenzen der Interdisziplinarität. Paradigmata. Zeitschrift für Menschen und Diskurse H. 2: 6-12.

Aus der Entwicklungsländer-Forschung 1960. Soziale Welt (11): 161-162.

Bahrdt, Hans Paul, Helmut Krauch und Horst Rittel 1960. Die wissenschaftliche Arbeit in Gruppen. Kölner Zeitschrift für Soziologie und Sozialpsychologie (12): 1-40.

Balsiger, Philipp W. 2005. Transdisziplinarität. Systematisch-vergleichende Untersuchung disziplinenübergreifender Wissenschaftspraxis. München: Wilhelm Fink.

Bergsträsser, Arnold 1956. Amerikastudien als Problem der Forschung und Lehre. Jahrbuch für Amerikastudien (1): 8-14.

Bernhard, Patrick, Holger Nehring und Anne Rohstock 2014. Der Kalte Krieg im langen 20. Jahrhundert. Neue Ansätze, Befunde und Perspektiven. In: Patrick Bernhard und Holger 
Nehring. Den Kalten Krieg denken. Beiträge zur sozialen Ideengeschichte. Essen: Klartext: 1139.

Bittel, Heinz 1972. Die vorgefertigte Universität. Erinnerungen an die Vor- und Frühgeschichte einer Baustelle. In: Materialien zur Geschichte der Ruhr-Universität Bochum. Bauidee und Baugeschehen. Hg. im Auftrage des Vorstandes der „Gesellschaft der Freunde der RuhrUniversität Bochum e.V." Bochum 1972: 11-29.

Blanke, Gustav H. 1963. Rezension zu Sigmund Skard, The American Myth and the European Mind (1961). Jahrbuch für Amerikastudien (8): 303-305.

Blankenburg, Erhard, Wolfgang Kaupen, Rüdiger Lautmann und Frank Rotter 1972. Kritische Anmerkungen zur rechtssoziologischen Forschung und Argumentation. In: Rehbinder und Schelsky (Hg.): 600-602.

Bornemann, Ernst und Rosemarie Nave-Herz 1964. Verzeichnis von Einrichtungen der Sozialwissenschaften und ihrer Grenzgebiete in Westdeutschland und Westberlin. Soziale Welt (15): 273-288.

Braungart, Wolfgang 2009. „Epochale“ Architektur. Das Gebäude der Universität Bielefeld. In: Sonja Asal und Stephan Schlak (Hg.). Was war Bielefeld? Eine ideengeschichtliche Nachfrage. Göttingen: Wallstein Verlag: 36-63.

Brinkmann, Johannes 1974. Friedensforschung in Skandinavien. Soziale Welt (25): 118-142.

Bühler, Charlotte 1959. Der menschliche Lebenslauf als psychologisches Problem. Zweite völlig veränderte Auflage. Göttingen: Hogrefe.

Burrichter, Clemens 1963. Informationen. Soziale Welt (14): 360-384.

Cohen-Cole, Jamie 2014. The Open Mind. Cold War Politics and the Sciences of Human Nature. Chicago: The University of Chicago Press.

Converse, P. E. 2001. Centers for Advanced Study: International/Interdisciplinary. In: Neil J. Smelser und Paul Baltes (Hg.). International Encyclopedia of the Social \& Behavioral Sciences. Vol. 3: 1613-1615.

Czempiel, Ernst-Otto 1965. Die Entwicklung der Lehre von den internationalen Beziehungen. Politische Vierteljahresschrift (6): 270-290.

Danckwortt, Dieter 1964. Zur Pädagogischen Problematik internationaler Seminare im Rahmen der Entwicklungshilfe. International Review of Education (10): 460-463.

Den Menschen mobilisieren 1964. Auszüge aus Referaten der Travemünder Tagung der Gesellschaft für Wirtschafts- und Sozialwissenschaften. FAZ (03.10.): 5.

Der Minister für Landesplanung, Wohnungsbau und öffentliche Arbeiten des Landes NordrheinWestfalen (Hg.) 1965. Die Universität Bochum. Gesamtplanung. Stuttgart/Bern: K. Krämer.

Die Jahrbücher für Friedens- und Konfliktforschung 1978. Politische Vierteljahresschrift (19): 410411.

Die Universität Konstanz. Bericht des Gründungsausschusses (Juni 1965). In: Neuhaus (Hg.): Nr. 1339-Nr. 1471.

Edding, Friedrich 1965. Probleme der Bildungsökonomie. Weltwirtschaftliches Archiv (94): 1-4.

Eggers, Michael und Matthias Rothe 2009. Einleitung: Die Begriffsgeschichte ist tot, es lebe die Begriffsgeschichte! In: Dies. (Hg.). Wissenschaftsgeschichte als Begriffsgeschichte. Terminologische Umbrüche im Entstehungsprozess der modernen Wissenschaften. Bielefeld: transcript: 7-22.

Empfehlungen zum Aufbau der Universität Bochum. Denkschrift des Gründungsausschusses. Bochum (Dezember) 1962.

Empfehlungen zur Gründung der Universität Bochum. Denkschrift des Gründungsausschusses, veröffentlicht vom Kultusministerium des Landes Nordrhein-Westfalen Dezember 1962. In: Neuhaus (Hg.): Nr. 388-Nr. 571.

Engelhardt, Werner 1957. Grundprobleme der Einzelwirtschaftstypologie. Diss. Uni Köln.

Fischer, Wolfram 1962. Neuere Forschungen zur Wirtschafts- und Sozialgeschichte der USA. Vierteljahresschrift für Sozial- und Wirtschaftsgeschichte (49): 459-538.

Fischer, Wolfram 1963. Die Wirtschaftsgeschichte in den Vereinigten Staaten von Amerika. Zeitschrift für die gesamte Staatswissenschaft (119): 377-404.

Flohr, Heiner und Theo Stammen 1965. Politische Erziehung, Gemeinschaftskunde und Sozialwissenschaften. Zeitschrift für Politik, Neue Folge (12): 73-82.

Frank, Roberta 1988. „Interdisciplinary”. The First Half-Century. In: Eric G. Stanley und T. F. Hoad (Hg.). Words. For Robert Burchfield's Sixty-fifth Birthday. Cambridge: D. S. Brewer: 91-101. 
Franken, Joseph P. 1965. Bauidee und Gestalt der neuen Universität in Bochum. In: Hans Wenke und Joachim Knoll (Hg.). Festschrift zur Eröffnung der Universität Bochum. Bochum: F. Kamp: 25-56.

Freiräume für die Forschung? Round Table 2009. FRIAS News H. 1: 5-7.

Frenckner, T. Paulsson 1965. Europäische Gemeinschaftsforschung zur Entwicklung eines Lehrgebäudes der Unternehmensführung. Management International (5): 124-126.

Frick, Werner 2009. Editorial. FRIAS News H. 1: 3-4.

Frodeman, Robert, Julie Thompson Klein und Carl Mitcham (Hg.) 2010. The Oxford Handbook of Interdisciplinarity. Oxford: Oxford University Press.

Gäfgen, Gerard 1961. Zur Theorie kollektiver Entscheidungen in der Wirtschaft. Jahrbücher für Nationalökonomie und Statistik (173): 1-49.

Galison, Peter 1996. Introduction: The Context of Disunity. In: Peter Galison und David J. Stump (Hg.). The Disunity of Science. Boundaries, Contexts, and Power. Stanford: Stanford University Press: 1-33.

Gall, Alexander 2009. Konstruieren, kommunizieren, präsentieren. Zur Einführung. In: Ders. (Hg.). Bilder von Wissenschaft und Technik. Göttingen: Wallstein: 9-22.

Gethmann, Daniel und Susanne Hauser 2009. Einleitung. In: Dies. (Hg.): 9-5.

Gethmann, Daniel und Susanne Hauser (Hg.) 2009. Kulturtechnik Entwerfen. Praktiken, Konzepte und Medien in Architektur und Design Science. Bielefeld: transcript.

Geulen, Christian 2010. Plädoyer für eine Geschichte der Grundbegriffe des 20. Jahrhunderts. Zeithistorische Forschungen/Studies in Contemporary History, Online-Ausgabe, H. 1 (2010). URL: http://www.zeithistorische-forschungen.de/1-2010/id=4488 (20.01.2016), Druckausgabe: 79-97.

Giuliani, Luca 2015. Vorwort des Herausgebers. Wissenschaftskolleg zu Berlin. Jahrbuch (2013/14): 11-13. URL: http://www.wiko-berlin.de/fileadmin/Dateien_Redakteure/pdf/Jahrbuecher/ Wiko-JB-2013-2014.pdf (20.01.2016).

Göppinger, Hans E. 1966. Kriminologie als interdisziplinäre Wissenschaft. Kriminalbiologische Gegenwartsfragen (7) (Vorträge bei der XIII. Tagung der Kriminalbiologischen Gesellschaft vom 7. bis 10. Oktober 1965 in Gießen): 1-16.

Grotemeyer, Karl Peter 1975. Grußwort des Rektors der Universität zur Eröffnung der Ausstellung. In: Klaus Köpke et al. (Hg.). Universität Bielefeld. Bauen in der industriellen Welt. Dokumentation zur Architektur der Universität Bielefeld. Kunsthalle Bielefeld vom 19. Oktober bis 16. November 1975. Bielefeld: Kunsthalle Bielefeld: 7.

Gründungsausschuß der Universität im Ostwestfälischen Raum (1966). Strukturmerkmale der neuen Universität in Ostwestfalen. In: Neuhaus (Hg.): Nr. 364-Nr. 387.

Gutachtliche Stellungnahme des Preisgerichtes 1969. In: Herzog et al. (Hg.): 29-32.

Hallauer, Fridel 1990. Baugeschichte der Ruhr-Universität Bochum. Fakten und Wertungen nach 25 Jahren. In: Burkhard Dietz, Winfried Schulze und Wolfgang Weber (Hg.). Universität und Politik. Festschrift zum 25jährigen Bestehen der Ruhr-Universität Bochum, Bd 1. Bochum: Ruhr Universität Bochum: 201-241.

Henke, Christopher R. und Thomas F. Gieryn 2007. Sites of Scientific Practices. The Enduring Importance of Place. In: Edward J. Hackett (Hg.). Handbook of Science and Technology Studies. London: MIT Press: 353-376.

Herzog, Dietrich 1964. „Soziologie der Demokratie“ von Seymour Martin Lipset. Soziale Welt (15): 151-154.

Herzog, Helmut et al. (Hg.) 1969. Dokumentation und Erläuterung zur Entwicklung der mit dem ersten Preis ausgezeichneten Wettbewerbsarbeit für die Universität Bielefeld. Bielefeld. Landesgeschichtliche Bibliothek.

Heßler, Martina 2005. Bilder zwischen Kunst und Wissenschaft. Neue Herausforderungen für die Forschung. Geschichte und Gesellschaft (31): 266-292.

Heßler, Martina 2007. Die kreative Stadt. Zur Neuerfindung eines Topos. Bielefeld: Transcript.

Heyen, Erk Volkmar 1978. Zum Verhältnis von philosophischer und wissenschaftlicher Anthropologie. Archiv für Rechts- und Sozialphilosophie (64): 509-533.

Hilgendorf, Eric 2010. Bedingungen gelingender Interdisziplinarität - am Beispiel der Rechtswissenschaft. Juristenzeitung (65): 913-968.

Historisches Wörterbuch interdisziplinärer Begriffe (Zentrum für Literatur- und Kulturforschung Berlin), Stichwort „Interdisziplinärität“ 2015. URL: http://www.begriffsgeschichte.de/doku. php?id=interdisziplinaritaet (12.11.2015). 
Hoffmann, Stefan-Ludwig und Katrin Kollmeier 2012. Geschichtliche Grundbegriffe reloaded? Writing the Conceptual History of the Twentieth Century. Contributions to the History of Concepts (7) H. 1.

Holtorf, Christian 2014. Einheit und Differenz. Aporien der Interdisziplinarität. In: Schier und Schwinger (Hg.): 63-77.

Holzhey, Helmut 1976. Interdisziplinarität. In: Historisches Wörterbuch der Philosophie, Bd. 4. Basel: Schwabe \& Co.: 476-478.

Hoppe-Sailer, Richard, Cornelia Jöchner und Frank Schmitz 2015. Einführung. In: Dies. (Hg.): 911.

Hoppe-Sailer, Richard, Cornelia Jöchner und Frank Schmitz (Hg.) 2015. Ruhr-Universität Bochum. Architekturvision der Nachkriegsmoderne. Berlin: Mann Verlag.

Hübenthal, Ursula 1991. Interdisziplinäres Denken. Versuch einer Bestandsaufnahme und Systematisierung. Stuttgart: Steiner.

Interdisziplinarität 2013. FRIAS News H. 8: 22-23.

Jessen, Ralph und Jürgen John 2005. Editorial: Wissenschaft und Universitäten im geteilten Deutschland der 1960er Jahre. Jahrbuch für Universitätsgeschichte (8): 7-24.

Jüchter, Heinz Theodor 1963. Studienreform nach den Vorstellungen des Verbandes Deutscher Studentenschaften. FAZ (03.04.): 11.

Jungert, Michael 2010. Interdisziplinarität. Theorie, Praxis, Probleme. Darmstadt: Wissenschaftliche Buchgesellschaft.

Kade, Gerhard 1960. Rezension zu G. T. Guilbaud, What is Cybernetics, London-MelbourneToronto 1959. Jahrbücher für Nationalökonomie und Statistik (172): 443-444.

Kade, Gerhard 1962a. Rezension zu J. Restle, Psychology of Judgement and Choice u.a. Jahrbücher für Nationalökonomie und Statistik (174): 477-478.

Kade, Gerhard 1962b. Rezension zu Norbert Wiener, Cybernetics-or Control and Communication in the Animal and the Machine. Jahrbücher für Nationalökonomie und Statistik (174): 274-276.

Kade, Gerhard 1965. Rezension zu M. D. Mesarovic, Views on General Systems Theory. Jahrbücher für Nationalökonomie und Statistik (177): 550-551.

Kaiser, Karl 1975. Völkerrecht und Internationale Beziehungen. Zum Verhältnis zweier Wissenschaften. Die Friedens-Warte (58): 197-209.

Kaletsch H. et al. 1964. Vorgeschichte und Altertum. Historische Zeitschrift (198): 743-756.

Kandel, Isaac L. 1959. The Methodology of Comparative Education. International Review of Education (5): 270-280.

Kenkmann, Alfons 2000. Von der bundesdeutschen „Bildungsmisere“ zur Bildungsreform in den 60er Jahren. In: Axel Schildt, Karl Christian Lammers und Detlev Siegfried (Hg.). Dynamische Zeiten. Die 60er Jahre in beiden deutschen Gesellschaften. Hamburg: Christians: 402-423.

Kilcher, Andreas B. und Philipp Sarasin 2011. Editorial. Nach Feierabend. Zürcher Jahrbuch für Wissensgeschichte (7): 7-11.

Klein, Christa-Irene et al. 2014. Universität, Wissenschaft und Öffentlichkeit in Westdeutschland 1945 bis ca. 1970. Einleitung. In: Sebastian Brandt et al. (Hg.). Universität, Wissenschaft und Öffentlichkeit in Westdeutschland (1945 bis ca. 1970). Stuttgart: Franz Steiner: 7-38.

Kocka, Jürgen 2008. Disziplinen und Interdisziplinarität. In: Jürgen Reulecke und Volker Roelcke (Hg.). Wissenschaften im 20. Jahrhundert. Universitäten in der modernen Wissenschaftsgesellschaft. Stuttgart: Franz Steiner: 107-117.

Koselleck, Reinhart 2010 [1978]. Interdisziplinäre Forschung und Geschichtswissenschaft. In: Ders. Vom Sinn und Unsinn der Geschichte. Aufsätze und Vorträge aus vier Jahrzehnten. Berlin: Suhrkamp: 52-67.

Krämer, Sybille 2009. Operative Bildlichkeit. Von der „Grammatologie“ zu einer „Diagrammatologie“? Reflexionen über erkennendes „Sehen“. In: Martina Heßler und Dieter Mersch (Hg.). Logik des Bildlichen. Zur Kritik der ikonischen Vernunft. Bielefeld: transcript: 94-122.

Krippendorff, Ekkehart 1965. Profil einer Disziplin. Versuch über Herkunft und Stand der Politischen Wissenschaft in den Vereinigten Staaten. Politische Vierteljahresschrift (6): 184204.

Kröber, Günter 1983. Interdisziplinarität - ein aktuelles Erfordernis der Gesellschafts- und Wissenschaftsentwicklung. Deutsche Zeitschrift für Philosophie (31): 575-589.

Krüsselberg, Hans-Günter 1959. Kritische Betrachtungen zu G. Merks Aufsatz „Wahrscheinlichkeitstheorie und Investitionstheorie." Weltwirtschaftliches Archiv (82): 122-131. 
Kultusministerium Baden-Württemberg 1962. Denkschrift über die Errichtung von wissenschaftlichen Hochschulen in Baden-Württemberg. Stuttgart.

Kyle, Barbara 1959. Probleme sozialwissenschaftlicher Dokumentation. Soziale Welt (10): 213226.

Langewiesche, Dieter 2005. Einsamkeit und Gespräch. Hoffnungen eines Geisteswissenschaftlers bei der Gründung des Max-Weber-Kollegs. In: Hans Joas und Hans G. Kippenberg (Hg.). Interdisziplinarität als Lernprozeß. Erfahrungen mit einem handlungstheoretischen Forschungsprogramm. Göttingen: Wallstein: 13-21.

Larivière, Vincent und Yves Gingras 2014. Measuring Interdisciplinarity. In: Blaise Cronin und Cassidy R. Sugimoto (Hg.). Beyond Bibliometrics. Harnessing Multidimensional Indicators of Scholarly Impact. Cambridge Mass./London: MIT Press: 187-200.

Lauwerys, J. A. und Saul Robinsohn 1963. Einleitung zu der Sammlung von Referaten, die auf der Amsterdamer Konferenz der Comparative Education Society in Europe gehalten wurden. International Review of Education (9): 129-133.

Löning, Martin et al. 2011. Wie gründet man Universitäten? Helmut Schelskys Konzept und der gelungene Start der Universität Bielefeld. Dokumentation einer Ausstellung des Universitätsarchivs Bielefeld. Bielefeld: Universitätsarchiv.

Lundgreen, Peter (Hg.) 1994. Reformuniversität Bielefeld. Zwischen Defensive und Innovation. Bielefeld: Verlag für Regionalgeschichte.

Max-Planck-Institut für biologische Kybernetik 1968. Die Naturwissenschaften (55): 11.

Mikat, Paul und Helmut Schelsky (Hg.) 1966. Grundzüge einer neuen Universität. Zur Planung einer Hochschulgründung in Ostwestfalen. Gütersloh: Bertelsmann.

Mittelstraß, Jürgen 2003. Transdisziplinarität - wissenschaftliche Zukunft und institutionelle Wirklichkeit. Konstanzer Universitätsreden, Nr. 214. Konstanz.

Moran, Joe 2010. Interdisciplinarity. 2nd edition. London/New York: Routledge.

Mößner, Nicola 2012. Zur Einführung. Visualisierung und Erkenntnis. In: Dimitri Liebsch und Nicola Mößner (Hg.). Visualisierung und Erkenntnis. Bildverstehen und Bildverwenden in Natur- und Geisteswissenschaften. Köln: Herbert von Halem: 9-30.

Mühlenberg, Friedrich 1972. Methodenfragen zu interdisziplinären Forschungsansätzen. Die interdisziplinäre Länderanalyse. Africa Spectrum (7): 5-26.

Müller, Ernst und Falko Schmieder 2008. Einleitung. In: Dies. (Hg.). Begriffsgeschichte der Naturwissenschaften. Zur historischen und kulturellen Dimension naturwissenschaftlicher Konzepte. Berlin: de Gruyter: XI-XXIII.

Muthesius, Stefan 2000. The Postwar University. Utopianist Campus and College. New Haven: Yale University Press.

Nadler, Gerald 1964. Die neuen Aufgaben des Industrial Engineering. Management International (4) H. 1: 19-23.

Nail, Norbert 1975. Rezension zu „Zum Öffentlichen Sprachgebrauch in der Bundesrepublik Deutschland und in der DDR“. Zeitschrift für Dialektologie und Linguistik (42): 205-217.

Neuhaus, Rolf (Hg.) 1968. Dokumente zur Gründung neuer Hochschulen. Anregungen des Wissenschaftsrates. Empfehlungen und Denkschriften auf Veranlassung von Ländern in der Bundesrepublik Deutschland in den Jahren 1960-1966. Wiesbaden: Steiner.

Nickelsen, Kärin 2014. Kooperation und Konkurrenz in den Naturwissenschaften. In: Ralph Jessen (Hg.). Konkurrenz in der Geschichte. Praktiken - Werte - Institutionalisierungen. Frankfurt am Main/New York: Campus: 353-379.

Papenkort, Ulrich 1998. Studium Generale. In: Historisches Wörterbuch der Philosophie, Bd. 10: 351-352.

Paul, Helmut 1955. Das Studium der Sozialpsychologie an der Columbia-Universität. Soziale Welt (6): 380-381.

Paul, Helmut 1957. Das Büro für angewandte Sozialforschung an der Columbia-Universität in New York. Soziale Welt (8): 269-271.

Paulus, Stefan 2010. Vorbild USA? Amerikanisierung von Universität und Wissenschaft in Westdeutschland 1945-1976. München: Oldenbourg.

Pfeffer, Karl Heinz 1963. Soziologie der Entwicklungsländer. Bedingungen und Möglichkeiten eines Beitrags der deutschen Soziologie zur Erforschung des modernen Orients. Soziale Welt (14): 33-37.

Pfeifer, Gottfried 1964. Bericht über ein deutsch-mexikanisches Forschungsprojekt. Investigaciones interdisciplinarias en la region Puebla-Tlaxcala. Geographische Zeitschrift (52): 128-151. 
Rehbinder, Manfred und Helmut Schelsky (Hg.) 1972. Zur Effektivität des Rechts. Jahrbuch für Rechtssoziologie und Rechtstheorie, Bd. 3. Düsseldorf: Bertelsmann Universitätsverlag.

Reinalter, Helmut 2011. Interdisziplinarität. In: Ders. und Peter J. Brenner (Hg.). Lexikon der Geisteswissenschaften. Sachbegriffe - Disziplinen - Personen, Wien/Köln/Weimar: Böhlau Verlag: 368-372.

Rheinberger, Hans-Jörg 2006. Rezente Wissenschaft und ihre Erforschung. Das Beispiel Molekularbiologie. Medizinhistorisches Journal (41): 187-199.

Rheinberger, Hans-Jörg 2007. Historische Epistemologie zur Einführung. Hamburg: Junius.

Robinsohn, Saul 1965. Rezension zu George Bereday, Comparative Methods in Education. International Review of Education (11): 232-234.

Rohstock, Anne 2010. Von der „Ordinarienuniversität" zur „Revolutionszentrale"? Hochschulreform und Hochschulrevolte in Bayern und Hessen 1957-1976. München: Oldenbourg.

Rohstock, Anne 2013. Bologna als amerikanisches Kind des Kalten Krieges. Westliche Universitäten zwischen institutionellem Erbe und Weltkultur, 1945-2011. In: Marc Fabian Buck und Marcel Kabaum (Hg.). Ideen und Realitäten von Universitäten. Berlin: Peter Lang: 199_ 220.

Rohwedder, Uwe 2011. SDS-Hochschuldenkschrift und VDS-Neugründungsgutachten. Zwei studentische Beiträge zum Reformdiskurs der 1960er Jahre. In: Rainer Pöppinghege und Dieter Klenke (Hg.). Hochschulreformen früher und heute? Autonomie oder gesellschaftlicher Gestaltungsanspruch. Köln: SH-Verlag: 162-176.

Röttger-Rössler, Birgit 2005. Gespräche nach der zweiten Flasche oder: Überlegungen zur sozialen und emotionalen Identitätskonstruktion von ZiF-Forschungs-gruppen-mit-gliedern in informellen Kommunikationskontexten am Beispiel ausgewählter Narrative. Zif-Mitteilungen (1) 2005. URL: http://www.uni-bielefeld.de/ZIF/Allgemeines/texte.html (22.05.2015).

Sarasin, Philipp 2011. Was ist Wissensgeschichte? Internationales Archiv für Sozialgeschichte der deutschen Literatur (36): 159-172.

Schauz, Désirée 2014. Wissenschaftspolitische Sprache als Gegenstand von Forschung und disziplinärer Selbstreflexion. Das Programm des Forschungsnetzwerks CASTI. Forum interdisziplinäre Begriffsgeschichte (3): 49-61.

Schauz, Désirée 2015. Wissenschaftsgeschichte und das Revival der Begriffsgeschichte. N.T.M. Zeitschrift für Geschichte der Wissenschaften, Technik und Medizin (23): 53-63.

Schelsky, Helmut 1966a. Das Zentrum für interdisziplinäre Forschung. Eine Denkschrift. In: Paul Mikat und Helmut Schelsky (Hg.): 71-87.

Schelsky, Helmut [1965] 1966b. Grundzüge einer neuen Universität. Eine Denkschrift. Zweite Fassung des Dokuments vom 24.02.1965, vom 17.08.1965. In: Paul Mikat und Helmut Schelsky (Hg.): 35-70.

Schelsky, Helmut 1972. Soziologiekritische Bemerkungen zu gewissen Tendenzen von Rechtssoziologen. In: Rehbinder und Schelsky (Hg.): 603-611.

Schier, Carmen und Elke Schwinger (Hg.) 2014. Interdisziplinarität und Transdisziplinarität als Herausforderung akademischer Bildung. Innovative Konzepte für die Lehre an Hochschulen und Universitäten. Bielefeld: transcript.

Schiffers, Heinrich 1964. Zur Veranstaltung des Wissenschafts-Ausschusses der „Deutschen Afrika-Gesellschaft" in Köln vom 2.-4. Januar 1964. Neues Afrika. Monatsschrift für Politik, Wirtschaft und Kultur im neuen Afrika (6): 33-35.

Schlaeger, Jürgen 2008. Interdisziplinarität. In: Ansgar Nünning (Hg.). Metzler Lexikon Literaturund Kulturtheorie. Ansätze - Personen - Grundbegriffe. Vierte, aktualisierte und erweiterte Auflage. Stuttgart: Metzler: 324-325.

Schlager, Claudia 2014. Von Stolper- und Meilensteinen im Feld der interdisziplinären Lehre. Kulturanalytische Perspektiven auf organisationale Herausforderungen am Beispiel des Projektes „Der Coburger Weg“. In: Schier und Schwinger (Hg.): 197-210.

Schlochauer, Hans-Jürgen 1958. Rezension zu „Current Legal Problems“. Archiv des Völkerrechts (7): 221-222.

Schmidtke, Oliver 2007. Die Architektur der Ruhr-Universität Bochum sowie der Universität Bielefeld und ihre Entsprechung im technokratischen Deutungsmuster von Wissenschaft. In: Andreas Franzmann und Barbara Wolbring (Hg.). Zwischen Idee und Zweckorientierung. Vorbilder und Motive von Hochschulreformen seit 1945. Berlin: Akademie Verlag: 137-182.

Siegert, Bernhard 2009. Weiße Flecken und finstre Herzen. Von der symbolischen Weltordnung zur Weltentwurfsordnung. In: Gethmann und Hauser (Hg.): 19-47. 
Sills, David L. 1986. A Note on the Origin of "Interdisciplinary". Items. Social Science Research Council (40): 17-18.

Söllner, Alfons 2013. Mehr Universität wagen! Helmut Schelsky und die Hochschulpolitik der 1960er Jahre. In: Alexander Gallus (Hg.). Helmut Schelsky - der politische Anti-Soziologe. Eine Neurezeption. Göttingen: Wallstein: 100-114.

Sondermann, Michael et al. (2008). Die Exzellenzinitiative. Institut für Forschungsinformation und Qualitätssicherung, Working Paper No. 5. URL: http://www.forschungsinfo.de/ publikationen/download/working_paper_5_2008.pdf (21.01.2016).

Sonnenhol, Gustav Adolf 1965. Die Ordnungspolitik der Entwicklungsländer. Wirtschaftspolitische Chronik (18): 17-44.

Sprenger, Gerhard und Peter Weingart 1994. Zentrum für interdisziplinäre Forschung. In: Peter Lundgreen (Hg.): 397-410.

Stachowiak, Herbert 1957. Über kausale, konditionale und strukturelle Erklärungsmodelle. Philosophia Naturalis (4): 403-433.

Städtebaulicher Ideenwettbewerb für die Gestaltung der Universität Bochum i. W. 1963. Die Bauverwaltung (14): 272-277.

Stammer, Otto 1961. Aspekte der Totalitarismusforschung. Soziale Welt (12): 97-111.

Steinmetz, Willibald 2012. Some Thoughts on a History of Twentieth-Century German Basic Concepts. In: Hoffmann und Kollmeier (Hg.): 87-100.

Stöhr, Rudolf W. 1962. Das Studium der Unternehmensführung in Europa und in den Vereinigten Staaten. Management International (2): 21-24.

Stüer, Franz [1963] 1965. Grundlagen der Gesamtplanung: Programmgestaltung. In: Der Minister für Landesplanung, Wohnungsbau und öffentliche Arbeiten des Landes NordrheinWestfalen (Hg.): 25-37.

Thompson Klein, Julie 1990. Interdisciplinarity. History, Theory, \& Practice. Detroit: Wayne State University Press.

Tichy, F. 1964. Bericht über den Beginn geographischer Arbeiten im Rahmen der deutschmexikanischen interdisziplinären Forschungen im Becken von Puebla-Tlaxcala. Geographische Zeitschrift (52): 151-157.

Tonutti, Emil 1977. Das Gründungskonzept der Medizinisch-Naturwissenschaftlichen Hochschule Ulm (1964 bis 1967). In: 10 Jahre Universität Ulm. Ansprachen, Referate und LudwigHeilmeyer-Gedächtnisvorlesung aus Anlaß des 10jährigen Bestehens der Universität Ulm im Februar 1977. Ulm: Pressestelle der Universität: 12-16.

Trippe, Dean 2015. Illustration. Nature (525): 305.

Über die Gründung einer Universität zu Bremen. Denkschrift, vorgelegt der Universitätskommission des Senats der Freien Hansestadt Bremen von Dr. Hans Werner Rothe 1960. In: Neuhaus (Hg.), Nr. 595-1139.

Universität Bielefeld (Hg.) 1974. Bauplanung der Universität Bielefeld. Bielefeld: Landesgeschichtliche Bibliothek Bielefeld.

Veit-Brause, Irmline 2000. Die Interdisziplinarität der Begriffsgeschichte als Brücke zwischen den Disziplinen. Archiv für Begriffsgeschichte (Sonderheft), hg. von Gunter Scholz. Hamburg: Meiner: 15-29.

Verband Deutscher Studentenschaften 1962. Studenten und die neue Universität. Gutachten einer Kommission des Verbandes Deutscher Studentenschaften zur Neugründung von Wissenschaftlichen Hochschulen. Bonn.

Voßkamp, Wilhelm 1984. Von der wissenschaftlichen Spezialisierung zum Gespräch zwischen den Disziplinen. In: Herbert Wendt und Norbert Loacker (Hg.). Kindlers Enzyklopädie Der Mensch. Band 7. Zürich: Kindler: 445-462

von Cube, Alexandra 1992. Die Ruhr-Universität Bochum. Bauaufgabe - Baugeschichte Baugedanke. Eine kunsthistorische Untersuchung. Bochum: Brockmeyer.

von Hentig, Hartmut 1971. Interdisziplinarität, Wissenschaftsdidaktik, Wissenschaftspropädeutik. Merkur (25): 855-871.

von Mann, Wenzel Ritter 1970. Planungsbericht 1970 des Universitätsbauamtes. Konstanzer Blätter für Hochschulfragen (8): 12-64.

Vowinckel, Annette 2014. „Ich fürchte mich vor den Organisationslustigen“. Ein Dialog zwischen Hans Blumenberg und Reinhart Koselleck. Merkur (68): 546-550.

Wander, Hilde 1965. Rezension zu Demographic and Economic Change in Developed Countries, Princeton 1960. Weltwirtschaftliches Archiv (94): 32-34. 
Weingart, Peter 1995. Grenzüberschreitungen in der Wissenschaft. Crossing Boundaries in Science. Festschrift zum 25jährigen Bestehen des ZiF. Baden-Baden: Nomos.

Weingart, Peter 2002. Interdisziplinarität. Zwischen wissenschaftspolitischer Modefloskel und pragmatischem Förderkonzept. In: Impulse geben - Wissen stiften. 40 Jahre VolkswagenStiftung. Göttingen: Vandenhoeck \& Ruprecht: 159-195.

Weingart, Peter und Nico Stehr (Hg.) 1999. Practising Interdisciplinarity. Toronto: University of Toronto Press.

Weisner, Ulrich 1994. Die Architektur der Universität. In: Lundgreen (Hg.): 487-497.

Winiwarter, Verena 2014. Wege finden, beteiligt zu sein. In: Gert Dressel, Wilhelm Berger, Katharina Heimerl und Verena Winiwarter (Hg.). Interdisziplinär und transdisziplinär forschen. Praktiken und Methoden. Bielefeld: transcript: 11-12.

Winsemius, Willem 1960. Internationale Gemeinschaftsforschung über den „menschlichen Faktor" bei Unfällen in der Industrie. Soziale Welt (11): 134-150.

Wise, Norton M. 2006. Making Visible. Isis (97): 75-82.

Wittmann, Barbara 2012. Papierprojekte. Die Zeichnung als Instrument des Entwurfs. Zeitschrift für Medien- und Kulturforschung (3): 135-150.

Zum Geleit 1947. Studium Generale (1): 1-2.

Zweite Loccumer Reform-Tagung zur Juristenausbildung 1970. Zeitschrift für Rechtspolitik (3): $19-21$.

\section{Susanne Schregel}

The Institute for Advanced Studies in the

Humanities (IASH)

The University of Edinburgh

Hope Park Square

Edinburgh EH8 9NW

Scotland, UK

a.r.t.e.s. Graduate School for the Humanities

Cologne

Research Lab

Research Group “Transformations of Knowledge”

Universität zu Köln

Albertus-Magnus-Platz Cologne

Germany

E-Mail: s.schregel@uni-koeln.de 